\title{
A PSEUDOSPECTRAL QUADRATURE METHOD FOR NAVIER-STOKES EQUATIONS ON ROTATING SPHERES
}

\author{
M. GANESH, Q. T. LE GIA, AND I. H. SLOAN
}

\begin{abstract}
In this work, we describe, analyze, and implement a pseudospectral quadrature method for a global computer modeling of the incompressible surface Navier-Stokes equations on the rotating unit sphere. Our spectrally accurate numerical error analysis is based on the Gevrey regularity of the solutions of the Navier-Stokes equations on the sphere. The scheme is designed for convenient application of fast evaluation techniques such as the fast Fourier transform (FFT), and the implementation is based on a stable adaptive time discretization.
\end{abstract}

\section{INTRODUCTION}

In this paper we develop a pseudospectral quadrature method for the surface Navier-Stokes partial differential equations (PDEs) on the rotating unit sphere. Whereas the finite element method is best suited for handling nonsmooth processes, the spectral global basis computer models are very efficient and perform extremely well for processes with smooth regularity. For example, exponential convergence properties of the global Fourier basis spectral Galerkin methods (without quadrature) for the Ginzburg-Landau and Navier-Stokes PDEs on two-dimensional periodic cells are based on the Gevrey regularity of solutions of the PDEs [7, 20].

The complex three-dimensional flows in the atmosphere and oceans are considered to be accurately modeled by the Navier-Stokes PDEs of fluid mechanics together with classical thermodynamics [22]. Difficulties in computer modeling in these PDEs resulted in several simplified models for which spectral approximations are well known [2, 15, 22]. A famous open problem is to prove the global regularity for the three-dimensional incompressible Navier-Stokes PDEs 26. However, the precise Gevrey regularity of the unique solution of the (practically relevant) surface Navier-Stokes PDEs on the rotating sphere was proved in [5]. (Because the Earth's surface is an approximate sphere, a standard surface model, to study global atmospheric circulation on large planets, is the sphere.)

Consequently, a natural next step is to describe, analyze, and implement an exponentially converging pseudospectral method for the Navier-Stokes PDEs on the rotating sphere. In addition to the continuous model regularity results in [5], this paper is also motivated by the recent work [12, where discrete computer modeling of the Navier-Stokes PDEs on one-dimensional and toroidal domains [8] was extended to the unit sphere.

Received by the editor May 13, 2009 and, in revised form, April 18, 2010.

2010 Mathematics Subject Classification. Primary 65M12; Secondary 76D05.

Key words and phrases. Navier-Stokes equations, unit sphere, vector spherical harmonics. 
This paper is concerned with both implementation of our algorithm and its numerical analysis. The main numerical analysis contributions compared to results in [5], for the continuous problem, and in [12], for a discrete problem, are as follows.

For the spatially discrete pseudospectral quadrature Galerkin solutions of the Navier-Stokes equations, we prove (i) the stability (that is, uniform boundedness of approximate solutions, independent of their truncation parameter $N$ ); (see Theorem 4.1); and (ii) a spectrally accurate rate of convergence [that is, $\mathcal{O}\left(N^{-2 s}\right)$ accuracy, with $s$ depending on the smoothness of input data]; see Theorem 4.2 . We achieve these results by first generalizing the main regularity result in [5] to complex valued times (see Theorems 2.2 and 2.3), and then using this to prove the spectral rate of convergence of the time derivative of a Stokes projection comparison function (see Theorem 3.2). This time-derivative error result plays a crucial role in the proof of spectral convergence of the approximate solutions (see the proof of Theorem 4.2). We note that the main result in [12, page 978] establishes only convergence of the semi-discrete Galerkin method without quadrature in $L^{p}$ norms, but does not establish either stability or rate of convergence of the scheme.

The rate of convergence results, supported by numerical experiments, formed the core part of research on the Navier-Stokes equations on two-dimensional domains over the last few decades; see [8, 9, 27, and references therein. There is a vast literature on numerical methods and analysis for the Navier-Stokes PDE on bounded Euclidean domains (see [8, 9, 27] and references therein), but their counterparts on closed manifolds are rarer (see 12 and references therein). The implementation of the scheme in [12, page 978] is based on a fixed time-step explicit Runge-Kutta method that has a small stability region for the systems of ordinary differential equations arising from the spatial discretization.

The outline of this paper is as follows. In the next section, we recall various known preliminary results associated with the Navier-Stokes PDEs on the unit sphere, in strong and weak form. In Section 3, we introduce essential computational and numerical analysis tools required for the discretization and analysis of the Navier-Stokes equations. In Section 4, we describe and prove spectral accuracy of a pseudospectral quadrature method and give implementation details required to apply the FFT and adaptive-in-time simulation of the Navier-Stokes equations. In Section 5, we demonstrate computationally the accuracy and applicability of the algorithm for well-known benchmark examples.

\section{NAVier-Stokes EquATIONS On The Rotating Unit SPHERE}

The surface Navier-Stokes equations (NSE) describing a tangential, incompressible atmospheric stream on the rotating two-dimensional unit sphere $S \subset \mathbb{R}^{3}$ can be written as follows [5, 16, 17, 19, 28]:

$$
\begin{aligned}
\frac{\partial}{\partial t} \mathbf{u}+\boldsymbol{\nabla}_{\mathbf{u}} \mathbf{u}-\nu \boldsymbol{\Delta} \mathbf{u}+\boldsymbol{\omega} \times \mathbf{u}+\frac{1}{\rho} \mathbf{G r a d} p & =\mathbf{f}, \\
\operatorname{Div} \mathbf{u} & =0,\left.\quad \mathbf{u}\right|_{t=0}=\mathbf{u}_{0} \quad \text { on } S .
\end{aligned}
$$

Here $\mathbf{u}=\mathbf{u}(\widehat{\mathbf{x}}, t)=\left(u_{1}(\widehat{\mathbf{x}}, t), u_{2}(\widehat{\mathbf{x}}, t), u_{3}(\widehat{\mathbf{x}}, t)\right)^{T}$ is the unknown tangential divergencefree velocity field at $\widehat{\mathbf{x}} \in S$ and $t \in[0, T], p=p(\widehat{\mathbf{x}}, t)$ is the unknown pressure. The known components in (2.1) are the constant viscosity and density of the fluid, respectively denoted by $\nu, \rho$, the normal vector field $\boldsymbol{\omega}=\boldsymbol{\omega}(\widehat{\mathbf{x}})=\omega(\widehat{\mathbf{x}}) \widehat{\mathbf{x}}$ for the Coriolis acceleration term, and the external flow driving vector field $\mathbf{f}=\mathbf{f}(\widehat{\mathbf{x}}, t)$. 
The Coriolis function $\omega$ is given by $\omega(\widehat{\mathbf{x}})=2 \Omega \cos \theta$, where $\Omega$ is the angular velocity of the rotating sphere, and $\theta$ is the angle between $\widehat{\mathbf{x}}$ and the North Pole. The vorticity of the flow associated with the NSE (2.1), in the curvilinear coordinate system, is a normal vector field, defined, for a fixed $t \geq 0$, by

$$
\operatorname{Vort} \mathbf{u}(\widehat{\mathbf{x}}, t)=\operatorname{Curl}_{\widehat{\mathbf{x}}} \mathbf{u}(\widehat{\mathbf{x}}, t)=\widehat{\mathbf{x}} \Delta \Psi(\widehat{\mathbf{x}}, t), \quad \widehat{\mathbf{x}} \in S,
$$

for some scalar-valued vorticity stream function $\Psi$.

All spatial derivative operators in (2.1) and (2.2) are surface differential operators, obtained by restricting the corresponding domain operators (defined in a neighborhood of $S$ ) to the unit sphere, using standard differential geometry concepts on closed manifolds in $\mathbb{R}^{3}[16,17$.

Using the fact that the outward unit normal at $\widehat{\mathbf{x}} \in S$ is $\widehat{\mathbf{x}}$, the Curl of a scalar function $v$, of a normal vector field $\mathbf{w}=w \widehat{\mathbf{x}}$, and of a tangential vector field $\mathbf{v}$ on $S$ are, respectively, defined by

$\operatorname{Curl} v=-\widehat{\mathbf{x}} \times \operatorname{Grad} v, \quad \operatorname{Curl} \mathbf{w}=-\widehat{\mathbf{x}} \times \operatorname{Grad} w, \quad \operatorname{Curl}_{\widehat{\mathbf{x}}} \mathbf{v}=-\widehat{\mathbf{x}} \operatorname{Div}(\widehat{\mathbf{x}} \times \mathbf{v})$.

The surface diffusion operator acting on tangential vector fields on $S$ is denoted by $\boldsymbol{\Delta}$ (known as the Laplace-Beltrami or Laplace-de Rham operator) and is defined as

$$
\boldsymbol{\Delta} \mathbf{v}=\operatorname{Grad} \operatorname{Div} \mathbf{v}-\operatorname{CurlCurl}_{\widehat{\mathbf{x}}} \mathbf{v} .
$$

The following relations connecting the above operators will be used throughout the paper:

$$
\begin{aligned}
\operatorname{Div} \operatorname{Curl} v & =0, \quad \operatorname{Curl}_{\widehat{\mathbf{x}}} \operatorname{Curl} v=-\widehat{\mathbf{x}} \Delta v, \quad \Delta \operatorname{Curl} v=\operatorname{Curl} \Delta v, \\
2 \boldsymbol{\nabla}_{\mathbf{w}} \mathbf{v}= & -\operatorname{Curl}(\mathbf{w} \times \mathbf{v})+\operatorname{Grad}(\mathbf{w} \cdot \mathbf{v})-\mathbf{v D i v} \mathbf{w}+\mathbf{w D i v} \mathbf{v} \\
& -\mathbf{v} \times \operatorname{Curl}_{\widehat{\mathbf{x}}} \mathbf{w}-\mathbf{w} \times \operatorname{Curl}_{\widehat{\mathbf{x}}} \mathbf{v} .
\end{aligned}
$$

In particular, for tangential divergence-free vector fields, such as the solution $\mathbf{u}$ of the NSE, using (2.6), the nonlinear term in (2.1) can be written as

$$
\nabla_{\mathbf{u}} \mathbf{u}=\operatorname{Grad} \frac{|\mathbf{u}|^{2}}{2}-\mathbf{u} \times \operatorname{Curl}_{\widehat{\mathbf{x}}} \mathbf{u} .
$$

2.1. A weak formulation. A standard technique for removing the scalar pressure field from the Navier-Stokes equations is to multiply the first equation in (2.1) by test functions $\mathbf{v}$ from a space with elements having properties of the unknown velocity field $\mathbf{u}$ (in particular, Div $\mathbf{v}=0$ ) and then integrate to obtain a weak formulation. (The unknown $p$, can then be computed by solving a pressure Poisson equation, obtained by applying the surface divergence operator in (2.1).)

To this end, we introduce the standard inner products on the space of all square integrables: (i) scalar functions on $S$, denoted by $L^{2}(S)$; and (ii) tangential vector fields on $S$, denoted by $L^{2}(T S)$ :

$$
\begin{array}{ll}
\left(v_{1}, v_{2}\right)=\left(v_{1}, v_{2}\right)_{L^{2}(S)}=\int_{S} v_{1} \overline{v_{2}} d S, & v_{2}, v_{2} \in L^{2}(S), \\
\left(\mathbf{v}_{1}, \mathbf{v}_{2}\right)=\left(\mathbf{v}_{1}, \mathbf{v}_{2}\right)_{L^{2}(T S)}=\int_{S} \mathbf{v}_{1} \cdot \overline{\mathbf{v}_{2}} d S, & \mathbf{u}, \mathbf{v} \in L^{2}(T S),
\end{array}
$$

where $d S=\sin \theta d \theta d \phi$. Throughout the paper, the induced norm on $L^{2}(T S)$ is denoted by $\|\cdot\|$ and for other inner product spaces, say $X$ with inner product $(\cdot, \cdot)_{X}$, the associated norm is denoted by $\|\cdot\|_{X}$. For example, for $s>0$, standard 
norms in the scalar and vector-valued function Sobolev spaces $H^{s}(S)$ and $H^{s}(T S)$ are denoted by $\|\cdot\|_{H^{s}(S)}$ and $\|\cdot\|_{H^{s}(T S)}$, respectively. Since $H^{0}(T S)=L^{2}(T S)$, $\|\cdot\|_{H^{0}(T S)}=\|\cdot\|$.

We have the following identities for appropriate scalar and vector fields 16. $(2.4)-(2.6)]$ :

$$
\begin{aligned}
(\mathbf{G r a d} \psi, \mathbf{v})=-(\psi, \operatorname{Div} \mathbf{v}), & (\mathbf{C u r l} \psi, \mathbf{v})=\left(\psi, \operatorname{Curl}_{\widehat{\mathbf{x}}} \mathbf{v}\right), \\
\left(\mathbf{C u r l}_{\mathbf{C u r l}} \mathbf{\mathbf { x }} \mathbf{w}, \mathbf{z}\right)= & \left(\operatorname{Curl}_{\widehat{\mathbf{x}}} \mathbf{w}, \operatorname{Curl}_{\widehat{\mathbf{x}} \mathbf{z}} \mathbf{z}\right) .
\end{aligned}
$$

In (2.10), the $L^{2}(T S)$ inner product is used on the left-hand side and the $L^{2}(S)$ inner product is used on the right-hand side. Throughout the paper, we identify a normal vector field $\mathbf{w}$ with a scalar field $w$ and hence

$$
(\psi, \mathbf{w}):=(\psi, w)_{L^{2}(S)}, \quad \mathbf{w}=\widehat{\mathbf{x}} w, \quad \psi, w \in L^{2}(S) .
$$

Using (2.5), smooth $\left(C^{\infty}\right)$ tangential fields on $S$ can be decomposed into two components, one in the space of all divergence-free fields and the other through the Hodge decomposition theorem [1:

$$
C^{\infty}(T S)=C^{\infty}(T S ; \text { Grad }) \oplus C^{\infty}(T S ; \text { Curl }),
$$

where

$$
\begin{aligned}
C^{\infty}(T S ; \mathbf{G r a d}) & =\left\{\operatorname{Grad} \psi: \psi \in C^{\infty}(S)\right\}, \\
C^{\infty}(T S ; \operatorname{Curl}) & =\left\{\operatorname{Curl} \psi: \psi \in C^{\infty}(S)\right\} .
\end{aligned}
$$

For $s \geq 0$, let $C^{\infty, s}\left(T S\right.$; Curl ) denote the closure of $C^{\infty}(T S ; \mathbf{C u r l})$ in the $H^{s}(T S)$ norm. In particular, following [5] we introduce a simpler notation:

$$
\begin{aligned}
& H=\text { closure of } C^{\infty}(T S ; \text { Curl }) \text { in } L^{2}(T S)=C^{\infty, 0}(T S ; \text { Curl }), \\
& V=\text { closure of } C^{\infty}(T S ; \text { Curl }) \text { in } H^{1}(T S)=C^{\infty, 1}(T S ; \text { Curl }) .
\end{aligned}
$$

Using the Gauss surface divergence theorem, for any scalar-valued function $v$ on $S$ with Grad $v \in L^{2}(T S)$, using (2.10), we have

$$
(\mathbf{G r a d} v, \mathbf{w})=\int_{S} \operatorname{Grad} v \cdot \overline{\mathbf{w}} d S=-\int_{S} v \cdot \operatorname{Div} \overline{\mathbf{w}} d S=0, \quad \mathbf{w} \in V,
$$

and hence the unknown pressure can be eliminated from the first equation in (2.1) through the weak formulation.

Following [16, Page 567], for the diffusion part of the NSE, we consider the Stokes operator

$$
\mathbf{A}=\operatorname{Curl~Curl}_{\widehat{\mathbf{x}}} .
$$

Using (2.4) and (2.5), it is easy to see that the Stokes operator is the restriction of the vector Laplace-de Rham operator $-\boldsymbol{\Delta}$ on $V ; \mathbf{A}=-\mathbf{P}_{\text {Curl }} \boldsymbol{\Delta}$, where $\mathbf{P}_{\text {Curl }}: L^{2}(T S) \rightarrow H$ is the orthogonal projection onto the divergence-free tangent space.

For each positive integer $L=1,2, \ldots$, the eigenvalue $\lambda_{L}$ and the corresponding eigenvectors of the Stokes operator $\mathbf{A}$ are given by

(2.17) $\lambda_{L}=L(L+1), \quad \mathbf{Z}_{L, m}(\theta, \varphi)=\lambda_{L}^{-1 / 2} \operatorname{Curl} Y_{L, m}(\theta, \varphi), \quad m=-L, \ldots, L$,

where $Y_{L, m}$ are the scalar orthonormal spherical harmonics of degree $L$, defined by

$$
Y_{L, m}(\theta, \varphi)=\left[\frac{(2 L+1)}{4 \pi} \frac{(L-|m|) !}{(L+|m|) !}\right]^{1 / 2} P_{L}^{m}(\cos \theta) e^{i m \varphi}, \quad m=-L, \ldots, L,
$$


with $P_{L}^{m}$ being the associated Legendre polynomials so that $\overline{Y_{L, m}}=(-1)^{m} Y_{L,-m}$.

The spectral property $\mathbf{A} \mathbf{Z}_{L, m}=\lambda_{L} \mathbf{Z}_{L, m}$ follows from the fact that $Y_{L, m}$ are eigenfunctions of the scalar Laplace-Beltrami operator $-\Delta$ with eigenvalues $\lambda_{L}$, the definition of the Stokes operator $\mathbf{A}$ in (2.16), $\mathbf{Z}_{L, m}$ in (2.17), (2.5), and (2.3). Since $\left\{Y_{L, m}: L=0,1, \ldots ; m=-L, \ldots, L\right\}$ is an orthonormal basis for $L^{2}(S)$, it is easy to see that $\left\{\mathbf{Z}_{L, m}: L=1, \ldots ; m=-L, \ldots, L\right\}$ is an orthonormal basis for $H$. Thus an arbitrary $\mathbf{v} \in H$ can be written as

$$
\mathbf{v}=\sum_{L=1}^{\infty} \sum_{m=-L}^{L} \widehat{\mathbf{v}}_{L, m} \mathbf{Z}_{L, m}, \quad \widehat{\mathbf{v}}_{L, m}=\int_{S} \mathbf{v} \cdot \overline{\mathbf{Z}_{L, m}} d S=\left(\mathbf{v}, \mathbf{Z}_{L, m}\right) .
$$

We consider a subset of $H$,

$$
\mathcal{D}\left(\mathbf{A}^{s / 2}\right)=\left\{\mathbf{v} \in H: \mathbf{v}=\sum_{L=1}^{\infty} \sum_{m=-L}^{L} \widehat{\mathbf{v}}_{L, m} \mathbf{Z}_{L, m}, \quad \sum_{L=1}^{\infty} \sum_{m=-L}^{L} \lambda_{L}^{s}\left|\widehat{\mathbf{v}}_{L, m}\right|^{2}<\infty\right\},
$$

which is the divergence-free subset of the Sobolev space $H^{s}(T S)$. For every $\mathbf{v} \in$ $\mathcal{D}\left(\mathbf{A}^{s / 2}\right)$, we set

$$
\|\mathbf{v}\|_{H^{s}(T S)}=\left[\sum_{L=1}^{\infty} \sum_{m=-L}^{L} \lambda_{L}^{s}\left|\widehat{\mathbf{v}}_{L, m}\right|^{2}\right]^{1 / 2}
$$

and for $\mathbf{v} \in \mathcal{D}\left(\mathbf{A}^{s / 2}\right)$, we define

$$
\mathbf{A}^{s / 2} \mathbf{v}:=\sum_{L=1}^{\infty} \sum_{m=-L}^{L} \lambda_{L}^{s / 2} \widehat{\mathbf{v}}_{L, m} \mathbf{Z}_{L, m} \in H
$$

For a tangential vector field $\mathbf{v}$ on $S$, we define the Coriolis operator $\mathbf{C}$ :

$$
(\mathbf{C v})(\widehat{\mathbf{x}})=\boldsymbol{\omega}(\widehat{\mathbf{x}}) \times \mathbf{v}(\widehat{\mathbf{x}})=\omega(\widehat{\mathbf{x}})(\widehat{\mathbf{x}} \times \mathbf{v}), \quad \omega(\widehat{\mathbf{x}})=2 \Omega \cos \theta .
$$

To treat the nonlinear term in (2.1), we consider the trilinear form $b$ on $V \times V \times V$, defined as

$$
b(\mathbf{v}, \mathbf{w}, \mathbf{z})=\left(\boldsymbol{\nabla}_{\mathbf{v}} \mathbf{w}, \mathbf{z}\right)=\int_{S} \boldsymbol{\nabla}_{\mathbf{v}} \mathbf{w} \cdot \overline{\mathbf{z}} d S, \quad \mathbf{v}, \mathbf{w}, \mathbf{z} \in V .
$$

Using (2.6) and (2.10), for divergence-free fields $\mathbf{v}, \mathbf{w}, \mathbf{z}$, the trilinear form can be written as

$$
b(\mathbf{v}, \mathbf{w}, \mathbf{z})=\frac{1}{2} \int_{S}\left[-\mathbf{v} \times \mathbf{w} \cdot \operatorname{Curl}_{\widehat{\mathbf{x}}} \overline{\mathbf{z}}+\operatorname{Curl}_{\widehat{\mathbf{x}}} \mathbf{v} \times \mathbf{w} \cdot \overline{\mathbf{z}}-\mathbf{v} \times \operatorname{Curl}_{\widehat{\mathbf{x}}} \mathbf{w} \cdot \overline{\mathbf{z}}\right] d S .
$$

Moreover [16, Lemma 2.1],

$$
b(\mathbf{v}, \mathbf{w}, \mathbf{w})=0, \quad b(\mathbf{v}, \mathbf{z}, \mathbf{w})=-b(\mathbf{v}, \mathbf{w}, \mathbf{z}) \quad \mathbf{v}, \mathbf{w}, \mathbf{z} \in V .
$$

Throughout the paper, the space $V$ is equipped with the norm $\|\cdot\|_{V}^{2}=(\mathbf{A} \cdot, \cdot)$.

Thus, using (2.4), (2.10), (2.16), and (2.25), a weak solution of the Navier-Stokes equations (2.1) is a vector field $\mathbf{u} \in L^{2}([0, T] ; V)$ with $\mathbf{u}(0)=\mathbf{u}_{0}$ that satisfies the weak form

$$
\left(\mathbf{u}_{t}, \mathbf{v}\right)+b(\mathbf{u}, \mathbf{u}, \mathbf{v})+\nu\left(\operatorname{Curl}_{\widehat{\mathbf{x}}} \mathbf{u}, \mathbf{C u r l}_{\widehat{\mathbf{x}}} \mathbf{v}\right)+(\mathbf{C u}, \mathbf{v})=(\mathbf{f}, \mathbf{v}), \quad \mathbf{v} \in V .
$$


This weak formulation can be written in operator equation form on $V^{*}$, the adjoint of $V$ : Let $\mathbf{f} \in L^{2}\left([0, T] ; V^{*}\right)$ and $\mathbf{u}_{0} \in H$. Find a vector field $\mathbf{u} \in L^{2}([0, T] ; V)$, with $\mathbf{u}_{t} \in L^{2}\left([0, T] ; V^{*}\right)$ such that

$$
\mathbf{u}_{t}+\nu \mathbf{A u}+\mathbf{B}(\mathbf{u}, \mathbf{u})+\mathbf{C u}=\mathbf{f}, \quad \mathbf{u}(0)=\mathbf{u}_{0},
$$

where the bilinear form $\mathbf{B}(\mathbf{u}, \mathbf{v}) \in V^{*}$ is defined by

$$
(\mathbf{B}(\mathbf{u}, \mathbf{v}), \mathbf{w})=b(\mathbf{u}, \mathbf{v}, \mathbf{w}) \quad \mathbf{w} \in V .
$$

In the subsequent error analysis, we need the following estimate for the nonlinear term (see Lemma 6.1 in Appendix):

$$
\left\|\mathbf{A}^{-\delta} \mathbf{B}(\mathbf{u}, \mathbf{v})\right\| \leq\left\{\begin{array}{l}
C\left\|\mathbf{A}^{1-\delta} \mathbf{u}\right\|\|\mathbf{v}\| \leq C\left\|\mathbf{A}^{1 / 2} \mathbf{u}\right\|\|\mathbf{v}\|, \\
C\|\mathbf{u}\|\left\|\mathbf{A}^{1-\delta} \mathbf{v}\right\| \leq C\|\mathbf{u}\|\left\|\mathbf{A}^{1 / 2} \mathbf{v}\right\|,
\end{array} \quad \delta \in(1 / 2,1) \quad \mathbf{u}, \mathbf{v} \in V .\right.
$$

In (2.30), as throughout the paper, $C$ is a generic constant independent of $\mathbf{u}$ and $\mathbf{v}$, (and the discretization parameter $N$ introduced in Section 3). From (2.30) we deduce the weak Lipschitz continuity property

$$
\begin{aligned}
\left\|\mathbf{A}^{-\delta}(\mathbf{B}(\mathbf{v}, \mathbf{v})-\mathbf{B}(\mathbf{w}, \mathbf{w}))\right\| \leq & C\|\mathbf{v}-\mathbf{w}\|, \\
& \delta \in(1 / 2,1), \quad \text { if }\left\|\mathbf{A}^{1 / 2} \mathbf{v}\right\|,\left\|\mathbf{A}^{1 / 2} \mathbf{w}\right\|<C .
\end{aligned}
$$

The existence and uniqueness of the solution $\mathbf{u} \in L^{2}([0, T] ; V)$ of the weak formulation (2.27) are discussed in [16, 17, 19. A regular solution of the Navier-Stokes equations (2.1) on $[0, T]$ is a tangential divergence-free velocity field $\mathbf{u}$ that satisfies the equation obtained by integrating in time the weak form (2.27), from $t_{0}$ to $t$, for almost every $t_{0}, t \in[0, T]$. In order to recall the existence, uniqueness, and Gevrey regularity of the regular solution, we need a few more additional details from $[5$. These are also needed as tools for analyzing our pseudospectral quadrature method.

2.2. Gevrey regularity of regular solution. The Gevrey class of functions of order $s>0$ and index $\sigma>0$, associated with the Stokes operator defined in (2.16), is denoted by $G_{\sigma}^{s / 2}$ and is defined as

$$
G_{\sigma}^{s / 2}:=\mathcal{D}\left(\mathbf{A}^{s / 2} e^{\sigma A^{1 / 2}}\right) \subset \mathcal{D}\left(\mathbf{A}^{s / 2}\right) .
$$

Using (2.20), the Gevrey space

$$
G_{\sigma}^{s / 2}=\left\{\mathbf{v} \in \mathcal{D}\left(\mathbf{A}^{s / 2}\right): \mathbf{v}=\sum_{L=1}^{\infty} \sum_{m=-L}^{L} \widehat{\mathbf{v}}_{L, m} \mathbf{Z}_{L, m}, \sum_{L=1}^{\infty} \sum_{m=-L}^{L} \lambda_{L}^{s} e^{2 \sigma \lambda_{L}^{1 / 2}}\left|\widehat{\mathbf{v}}_{L, m}\right|^{2}<\infty\right\}
$$

is a Hilbert space with respect to the inner product

$$
\langle\mathbf{v}, \mathbf{w}\rangle_{G_{\sigma}^{s / 2}}=\sum_{L=1}^{\infty} \sum_{m=-L}^{L} \lambda_{L}^{s} e^{2 \sigma \lambda_{L}^{1 / 2}} \widehat{\mathbf{v}}_{L, m}{\widehat{\widehat{\mathbf{w}}_{L, m}}}, \quad \mathbf{v}, \mathbf{w} \in G_{\sigma}^{s / 2} .
$$

First we recall the following result from [5, 29].

Theorem 2.1. If $\mathbf{u}_{0} \in \mathcal{D}\left(\mathbf{A}^{s+1 / 2}\right)$ and $\mathbf{f} \in L^{\infty}\left((0, \infty) ; \mathcal{D}\left(\mathbf{A}^{s} e^{\sigma_{1} \mathbf{A}^{1 / 2}}\right)\right)$, for some $s, \sigma_{1}>0$, then for all $t>0$ there exists $a T^{*}>0$, depending only on $\nu, \mathbf{f}$, and $\left\|\mathbf{A}^{s+1 / 2} \mathbf{u}_{0}\right\|_{L^{2}(T S)}$, such that the NSE (2.1) on $S$ have a unique regular solution $\mathbf{u}(\cdot, t)$ and $\mathbf{u}(\cdot, t) \in G_{\sigma(t)}^{s+1 / 2}$, where $\sigma(t)=\min \left\{t, T^{*}, \sigma_{1}\right\}$. 
In addition, from the assumption and proof of Theorem 2.1 [5, page 355],

$$
\left\|\mathbf{A}^{s+1 / 2} e^{\sigma(t) \mathbf{A}^{1 / 2}} \mathbf{u}(t)\right\|^{2} \leq M_{0}, \quad t>0,
$$

where $M_{0}$ depends on $\left\|\mathbf{A}^{s+1 / 2} \mathbf{u}(0)\right\|, \sup _{t>0}\left\|\mathbf{A}^{s} \mathbf{f}(\cdot, t)\right\|$ and $\nu$ but not on $t$. The bound in (2.35) is useful for establishing the quality of approximation of the Stokes projection of $\mathbf{u}$ in the next section (see Theorem 3.1). It is also convenient to have a similar bound for the time derivative of the Stokes projections with $t$ in (2.35) replaced with certain complex times $\zeta \in \mathbb{C}$, to prove the power of approximation of the time derivative of the Stokes projection (see Theorem 3.2). To this end, we consider the NSE extended to complex times $\zeta$,

$$
\frac{d \mathbf{u}}{d \zeta}+\nu \mathbf{A} \mathbf{u}+\mathbf{B}(\mathbf{u}, \mathbf{u})+\mathbf{C u}=\mathbf{f}, \quad \operatorname{Div} \mathbf{u}=0, \quad \mathbf{u}(0)=\mathbf{u}_{0}, \quad \zeta \in \mathbb{C}, \quad \text { on } S,
$$

with standard complexification (see 9]) of all the spaces and operators introduced earlier. In the next theorem, we extend arguments used in [9] for the solution of the NSE on the plane to the case of the sphere. The arguments differ in an essential way only for the nonlinear term.

Theorem 2.2. Let $\mathbf{u}_{0} \in \mathcal{D}\left(\mathbf{A}^{s+1 / 2}\right)$ and $\mathbf{f} \in C\left([0, T] ; \mathcal{D}\left(\mathbf{A}^{s} e^{\sigma_{1} \mathbf{A}^{1 / 2}}\right)\right)$, with $s \geq 1 / 4$. Let the domain $\mathbb{T}$ be defined by

$$
\mathbb{T}:=\left\{\zeta=r e^{i \theta}: 0 \leq r \leq T ;|\theta| \leq \pi / 4\right\} .
$$

We assume further that $\mathbf{f}(\cdot, \zeta)$ is analytic for $\zeta \in \mathbb{T}$ and that

$$
K:=\sup \left\{\left\|\mathbf{A}^{s} e^{\psi(r \cos \theta) \mathbf{A}^{1 / 2}} \mathbf{f}(\cdot, \zeta)\right\|^{2}: \zeta=r e^{i \theta} \in \mathbb{T}\right\}<\infty .
$$

Then there exists $T^{* *}>0$ such that

$$
\left\|\mathbf{A}^{s+1 / 2} e^{\psi(r \cos \theta) \mathbf{A}^{1 / 2}} \mathbf{u}(\zeta)\right\|^{2} \leq M_{1}, \quad \zeta \in \mathbb{T} \text { and }|\zeta| \leq T^{* *},
$$

where $M_{1}$ depends on $\left\|\mathbf{A}^{s+1 / 2} \mathbf{u}(0)\right\|$ and hence $\mathbf{u}(\cdot, \zeta) \in G_{\psi(r \cos \theta)}^{s+1 / 2}$, where $\psi(x):=\min \left\{x, T^{* *}, \sigma_{1}\right\}$.

Proof. Let $\zeta=r e^{i \theta}$ with $r>0$ and $|\theta| \leq \pi / 4$, and let

$$
\mathbf{u}_{\mathbf{A}}(\zeta):=\mathbf{A}^{s+1 / 2} e^{\psi(r \cos \theta) \mathbf{A}^{1 / 2}} \mathbf{u}(\zeta) .
$$

The definition of $\psi$ gives $\frac{d}{d x} \psi:=\psi^{\prime}(x) \leq 1$, and $\psi \leq \sigma_{1}$, thus for fixed $\theta$ we find

$$
\begin{aligned}
\frac{d}{d r} \mathbf{u}_{\mathbf{A}}(\zeta)= & \psi^{\prime}(r \cos \theta) \cos \theta \mathbf{A}^{s+1} e^{\psi(r \cos \theta) \mathbf{A}^{1 / 2}} \mathbf{u}(\zeta) \\
& +e^{i \theta} \mathbf{A}^{s+1 / 2} e^{\psi(r \cos \theta) \mathbf{A}^{1 / 2}} \frac{d \mathbf{u}}{d \zeta}(\zeta) .
\end{aligned}
$$

Using (2.39) in

$$
\frac{1}{2} \frac{d}{d r}\left\|\mathbf{u}_{\mathbf{A}}(\zeta)\right\|^{2}=\Re\left(\frac{d}{d r} \mathbf{u}_{\mathbf{A}}(\zeta), \mathbf{u}_{\mathbf{A}}(\zeta)\right),
$$

where $\Re(\cdot)$ denotes the real-part function, we get

$$
\begin{aligned}
\frac{1}{2} \frac{d}{d r}\left\|\mathbf{u}_{\mathbf{A}}(\zeta)\right\|^{2}= & \psi^{\prime}(r \cos \theta) \cos \theta \Re\left(\mathbf{A}^{1 / 2} \mathbf{u}_{\mathbf{A}}(\zeta), \mathbf{u}_{\mathbf{A}}(\zeta)\right) \\
& +\Re e^{i \theta}\left(\mathbf{A}^{s+1 / 2} e^{\psi(r \cos \theta) \mathbf{A}^{1 / 2}} \frac{d \mathbf{u}}{d \zeta}(\zeta), \mathbf{u}_{\mathbf{A}}(\zeta)\right) .
\end{aligned}
$$


Using (2.36) for the last term in (2.40) together with the fact that

$$
\left(\mathbf{A}^{s} e^{\psi(r \cos \theta) \mathbf{A}^{1 / 2}} \mathbf{C u}, \mathbf{A}^{s+1} e^{\psi(r \cos \theta) \mathbf{A}^{1 / 2}} \mathbf{u}\right)=0
$$

(see [5, Lemma 1]), we find

$$
\begin{aligned}
\frac{1}{2} \frac{d}{d r}\left\|\mathbf{u}_{\mathbf{A}}(\zeta)\right\|^{2}+\nu \cos \theta\left\|\mathbf{A}^{1 / 2} \mathbf{u}_{\mathbf{A}}(\zeta)\right\|^{2} \\
=\psi^{\prime}(r \cos \theta) \cos \theta\left(\mathbf{A}^{1 / 2} \mathbf{u}_{\mathbf{A}}, \mathbf{u}_{\mathbf{A}}\right)-\Re e^{i \theta}\left(\mathbf{A}^{s+1 / 2} e^{\psi \mathbf{A}^{1 / 2}} \mathbf{B}(\mathbf{u}, \mathbf{u}), \mathbf{u}_{\mathbf{A}}\right) \\
\quad+\Re e^{i \theta}\left(\mathbf{A}^{s} e^{\psi \mathbf{A}^{1 / 2}} \mathbf{f}, \mathbf{A}^{1 / 2} \mathbf{u}_{\mathbf{A}}\right),
\end{aligned}
$$

where in (2.41) and below we write $\mathbf{u}_{\mathbf{A}}=\mathbf{u}_{\mathbf{A}}(\zeta), \mathbf{u}=\mathbf{u}(\zeta), \mathbf{f}=\mathbf{f}(\zeta), \psi=\psi(r \cos \theta)$. From [5. Lemma 2], we have (with $p=\max \{2-s, 7 / 4\}=7 / 4$, since $s \geq 1 / 4$ ),

$$
\left|\left(\mathbf{A}^{s+1 / 2} e^{\psi \mathbf{A}^{1 / 2}} \mathbf{B}(\mathbf{u}, \mathbf{u}), \mathbf{u}_{\mathbf{A}}\right)\right| \leq C\left\|\mathbf{u}_{\mathbf{A}}\right\|^{3-p}\left\|\mathbf{A}^{1 / 2} \mathbf{u}_{\mathbf{A}}\right\|^{p} .
$$

Applying $\psi^{\prime} \leq 1$, the Cauchy-Schwarz inequality, (2.42) and Young's inequality $\left(a b \leq a^{q} / q+b^{q^{\prime}} / q^{\prime}\right.$ with $\left.1 / q+1 / q^{\prime}=1\right)$ with $q=2$ and $q=2 /(2-p)$ in (2.41), we get

$$
\begin{aligned}
& \frac{1}{2} \frac{d}{d r}\left\|\mathbf{u}_{\mathbf{A}}\right\|^{2}+\nu \cos \theta\left\|\mathbf{A}^{1 / 2} \mathbf{u}_{\mathbf{A}}\right\|^{2} \\
& \leq \cos \theta\left\|\mathbf{A}^{1 / 2} \mathbf{u}_{\mathbf{A}}\right\|\left\|\mathbf{u}_{\mathbf{A}}\right\|+C\left\|\mathbf{u}_{\mathbf{A}}\right\|^{3-p}\left\|\mathbf{A}^{1 / 2} \mathbf{u}_{\mathbf{A}}\right\|^{p}+\left\|\mathbf{A}^{s} e^{\psi \mathbf{A}^{1 / 2}} \mathbf{f}\right\|\left\|\mathbf{A}^{1 / 2} \mathbf{u}_{\mathbf{A}}\right\| \\
& \leq \frac{\nu \cos \theta}{4}\left\|\mathbf{A}^{1 / 2} \mathbf{u}_{\mathbf{A}}\right\|^{2}+\frac{\cos \theta}{\nu}\left\|\mathbf{u}_{\mathbf{A}}\right\|^{2} \\
&+C^{\frac{2}{2-p}} \frac{(2-p)}{p}\left(\frac{p}{\nu \cos \theta}\right)^{\frac{p}{2-p}}\left\|\mathbf{u}_{\mathbf{A}}\right\|^{\frac{2(3-p)}{2-p}}+\frac{\nu \cos \theta}{2}\left\|\mathbf{A}^{1 / 2} \mathbf{u}_{\mathbf{A}}\right\|^{2} \\
&+\frac{1}{\nu \cos \theta}\left\|\mathbf{A}^{s} e^{\psi \mathbf{A}^{1 / 2}} \mathbf{f}\right\|^{2}+\frac{\nu \cos \theta}{4}\left\|\mathbf{A}^{1 / 2} \mathbf{u}_{\mathbf{A}}\right\|^{2} .
\end{aligned}
$$

Therefore,

$$
\begin{aligned}
\frac{d}{d r}\left\|\mathbf{u}_{\mathbf{A}}\right\|^{2} \leq & \frac{2 \cos \theta}{\nu}\left\|\mathbf{u}_{\mathbf{A}}\right\|^{2}+C\left(\frac{1}{\nu \cos \theta}\right)^{\frac{p}{2-p}}\left\|\mathbf{u}_{\mathbf{A}}\right\|^{\frac{2(3-p)}{2-p}}+\frac{2}{\nu \cos \theta}\left\|\mathbf{A}^{s} e^{\psi \mathbf{A}^{1 / 2}} \mathbf{f}\right\|^{2} \\
\leq & \frac{2 \cos \theta}{\nu}\left(1+\left\|\mathbf{u}_{\mathbf{A}}\right\|^{2}\right)+C\left(\frac{1}{\nu \cos \theta}\right)^{\frac{p}{2-p}}\left(1+\left\|\mathbf{u}_{\mathbf{A}}\right\|^{2}\right)^{\frac{3-p}{2-p}} \\
& +\frac{2}{\nu \cos \theta}\left\|\mathbf{A}^{s} e^{\psi \mathbf{A}^{1 / 2}} \mathbf{f}\right\|^{2} .
\end{aligned}
$$

Using $|\theta| \leq \pi / 4$, and $\frac{3-p}{2-p}=5$, in (2.43), we get

$$
\frac{d}{d r}\left\|\mathbf{u}_{\mathbf{A}}\right\|^{2} \leq C\left(1+\left\|\mathbf{u}_{\mathbf{A}}\right\|^{2}\right)^{5}+\frac{2 \sqrt{2}}{\nu} K .
$$

With $|\theta| \leq \pi / 4$ fixed, let

$$
y(r)=1+\left\|\mathbf{u}_{\mathbf{A}}\right\|^{2} .
$$

Then by using (2.44) and $y \geq 1$ we obtain

$$
\frac{d}{d r} y \leq C y^{5},
$$

and hence by integrating the inequality we find that

$$
y(r) \leq 2 y(0)
$$


provided that

By setting

$$
0 \leq r \leq \frac{15}{64 C} \frac{1}{(y(0))^{4}}=\frac{15}{64 C} \frac{1}{\left(1+\left\|\mathbf{A}^{s+1 / 2} \mathbf{u}(0)\right\|^{2}\right)^{4}} .
$$

$$
T^{* *}:=\frac{15}{64 C} \frac{1}{\left(1+\left\|\mathbf{A}^{s+1 / 2} \mathbf{u}(0)\right\|^{2}\right)^{4}}, \quad M_{1}=1+2\left\|\mathbf{A}^{s+1 / 2} \mathbf{u}(0)\right\|^{2},
$$

we deduce that (2.38) holds for $0 \leq r \leq T^{* *}$.

We can extend the bound (2.38) to a larger domain which contains the interval $[0, T]$ using the following property of the NSE solution on the sphere [29]:

$$
\left\|\mathbf{A}^{s+1 / 2} \mathbf{u}(\cdot, t)\right\| \leq M_{2} \text { for all } t \in[0, T],
$$

where the constant $M_{2}$ depends only on $\left\|\mathbf{A}^{s+1 / 2} \mathbf{u}_{0}\right\|, \sup _{0 \leq t \leq T}\left\|\mathbf{A}^{s} \mathbf{f}(\cdot, t)\right\|$ and $\nu$ but not on $T$.

Theorem 2.3. Suppose $\mathbf{u}_{0}$ and $\mathbf{f}$ satisfy all the conditions in the domain $\mathbb{T}$ as in Theorem 2.2, Then

$$
\left\|\mathbf{A}^{s+1 / 2} e^{\psi(r \cos \theta) \mathbf{A}^{1 / 2}} \mathbf{u}(\zeta)\right\|^{2} \leq M_{3}, \quad \zeta \in \mathbb{T} \text { and }|\operatorname{Im} \zeta| \leq T^{* *} / \sqrt{2},
$$

where $M_{3}:=1+2 M_{2}^{2}, T^{* *}$ depends on $M_{2}$, and hence $\mathbf{u}(\cdot, \zeta) \in G_{\psi(r \cos \theta)}^{s+1 / 2}$ for all $\zeta \in \mathbb{T}$ with $|\operatorname{Im} \zeta| \leq T^{* *} / \sqrt{2}$.

Proof. We proceed as in the proof of Theorem 2.2 to obtain the ordinary differential equation

$$
\frac{d}{d r} y \leq C y^{5}
$$

where $y(r)=1+\left\|\mathbf{A}^{s+1 / 2} e^{\psi(r \cos \theta) \mathbf{A}^{1 / 2}} \mathbf{u}\left(r e^{i \theta}\right)\right\|^{2}$. By integrating the inequality we find that

provided that

$$
y(r) \leq 2 y(0)
$$

We define

$$
0 \leq r \leq \frac{15}{64 C} \frac{1}{\left(1+\left\|\mathbf{A}^{s+1 / 2} \mathbf{u}(0)\right\|^{2}\right)^{4}}
$$

$$
T_{1}(\rho):=\frac{15}{64 C} \frac{1}{\left(1+\rho^{2}\right)^{4}}, \quad \rho \geq 0 .
$$

If $T_{1}\left(\left\|\mathbf{A}^{s+1 / 2} \mathbf{u}(0)\right\|\right) \geq T$ we have finished the proof. Otherwise, we let

$$
T^{* *}=T_{1}\left(M_{2}\right),
$$

where $M_{2}$ is given in (2.45). For $\zeta=r e^{i \theta} \in \mathbb{T}, 0 \leq r \leq T^{* *}$,

$$
\left\|\mathbf{A}^{s+1 / 2} e^{\psi(r \cos \theta) \mathbf{A}^{1 / 2}} \mathbf{u}\left(r e^{i \theta}\right)\right\|^{2} \leq 1+2\left\|\mathbf{A}^{s+1 / 2} \mathbf{u}(0)\right\|^{2} .
$$

Consequently, (2.46) holds for $0 \leq r \leq T^{* *}$ with $M_{3}=1+2\left\|\mathbf{A}^{s+1 / 2} \mathbf{u}(0)\right\|^{2}$.

Next we consider the case $\zeta=T^{* *}+r e^{i \theta}$, with $r \in\left[0, T^{* *}\right]$. We define, for $|\theta| \leq \pi / 4$

$$
\mathbf{v}\left(r e^{i \theta}\right):=\mathbf{u}\left(T^{* *}+r e^{i \theta}\right), \quad r \in\left[0, T^{* *}\right] .
$$

Using $\left\|\mathbf{A}^{s+1 / 2} \mathbf{v}(0)\right\| \leq M_{2}$ we can apply the previous arguments to obtain (2.46) (with $\mathbf{u}$ replaced with $\mathbf{v}$ ) for $0 \leq r \leq T^{* *}$. We complete the proof and obtain the bound (2.46) by repeating the last argument $n$ times, where $n=\left\lceil T / T^{* *}\right\rceil$. 


\section{Finite dimensional SpaCES AND Stokes PROJECtions}

Throughout the remainder of the paper, with $s$ and $\sigma_{1}$ as in Theorem 2.1 and Theorem 2.2, we assume that

$$
\mathbf{u}_{0} \in \mathcal{D}\left(\mathbf{A}^{s+1 / 2}\right), \quad \mathbf{f} \in C\left([0, T] ; \mathcal{D}\left(\mathbf{A}^{s+1 / 2} e^{\sigma_{1} \mathbf{A}^{1 / 2}}\right)\right), \quad s \geq 1 / 4 .
$$

Natural finite dimensional spaces (depending on a parameter $N>0$ ) in which we seek approximations to $\mathbf{u}(t)$ are

$$
V_{N}:=\operatorname{span}\left\{\mathbf{Z}_{L, m}: L=1, \ldots, N ; m=-L, \ldots, L\right\} .
$$

The dimension of $V_{N}$ is $N^{2}+2 N$. Let $\boldsymbol{\Pi}_{N}: H \rightarrow V_{N}$ be the orthogonal projection with respect to the $L^{2}(T S)$ inner product defined by

$$
\boldsymbol{\Pi}_{N}(\mathbf{v})=\sum_{L=1}^{N} \sum_{m=-L}^{L} \widehat{\mathbf{v}}_{L, m} \mathbf{Z}_{L, m}
$$

Lemma 3.1. Let $\alpha>0$ be given. If $\mathbf{v} \in \mathcal{D}\left(\mathbf{A}^{\alpha / 2}\right)$, then

$$
\left\|\mathbf{v}-\Pi_{N} \mathbf{v}\right\| \leq N^{-\alpha}\|\mathbf{v}\|_{H^{\alpha}(T S)} .
$$

Proof. Using (2.17), (2.19), and (2.21) we get

$$
\begin{aligned}
\left\|\mathbf{v}-\boldsymbol{\Pi}_{N} \mathbf{v}\right\|^{2}=\sum_{L=N+1}^{\infty} \sum_{m=-L}^{L}\left|\widehat{\mathbf{v}}_{L, m}\right|^{2} & \leq N^{-2 \alpha} \sum_{L=N+1}^{\infty} \sum_{m=-L}^{L} \lambda_{L}^{\alpha}\left|\widehat{\mathbf{v}}_{L, m}\right|^{2} \\
& \leq N^{-2 \alpha}\|\mathbf{v}\|_{H^{\alpha}(T S)}^{2} .
\end{aligned}
$$

In particular, using (3.1), we get

$$
\left\|\mathbf{f}-\boldsymbol{\Pi}_{N} \mathbf{f}\right\| \leq N^{-(2 s+1)}\|\mathbf{f}\|_{H^{2 s+1}(T S)}, \quad t \in[0, T] .
$$

For computer implementation, the Fourier coefficients in (3.3) and all Galerkin type integrals in computational schemes for the NSE need to be approximated by cubature rules on the sphere, leading to a pseudospectral method. To this end, for a continuous scalar field $\psi$ on $S$, we consider a Gauss-rectangle quadrature sum $Q_{M}(\psi)$ with quadrature points $\left\{\widehat{\boldsymbol{\xi}}_{p, q}=\boldsymbol{p}\left(\theta_{p}, \phi_{q}\right)\right\}$ and positive weights $w_{p}$ of the form

$$
Q_{M}(\psi):=\frac{2 \pi}{M} \sum_{q=1}^{M} \sum_{p=1}^{M / 2} w_{p} \psi\left(\widehat{\boldsymbol{\xi}}_{p, q}\right)=\frac{2 \pi}{M} \sum_{q=1}^{M} \sum_{p=1}^{M / 2} w_{p} \psi\left(\theta_{p}, \phi_{q}\right),
$$

where $M \geq 2$ is an even integer, $w_{p}$ and $\cos \theta_{p}$ for $p=1, \ldots, M / 2$ are the GaussLegendre weights and nodes on $[-1,1]$ and $\phi_{q}=2 q \pi / M, q=1, \ldots, M$. The rule (3.6) is exact when $\psi$ is a polynomial of degree $M-1$ on $S$, that is,

$$
Q_{M} \psi=\int_{S} \psi d S, \quad \psi \in \mathcal{P}_{M-1}
$$

Hence, corresponding to (2.8) and (2.9), we define discrete inner products for scalar and vector fields on the unit sphere as

$$
\left(v_{1}, v_{2}\right)_{M}=Q_{M}\left(v_{1} v_{2}\right), \quad\left(\mathbf{v}_{1}, \mathbf{v}_{2}\right)_{M}=Q_{M}\left(\mathbf{v}_{1} \cdot \mathbf{v}_{2}\right) .
$$

The choice of $M$ is very important; we choose $M$ such that all Galerkin integrals with polynomial terms in our scheme are evaluated exactly. In particular, with the 
unknown tangential divergence-free velocity field sought in the polynomial space $V_{N}$, and knowing that the NSE nonlinearity is quadratic, we choose $M$ such that

$$
(\mathbf{B}(\mathbf{v}, \mathbf{w}), \mathbf{z})=(\mathbf{B}(\mathbf{v}, \mathbf{w}), \mathbf{z})_{M}, \quad \mathbf{v}, \mathbf{w}, \mathbf{z} \in V_{N}
$$

This holds, for example, if $M=3 N+2$. We define a computable counterpart of (3.3), using $\mathbf{L}_{N}: H \cap C(T S) \rightarrow V_{N}$, a discrete orthogonal projection with respect to the $M^{2} / 2$ point discrete inner product, as

$$
\begin{aligned}
\mathbf{L}_{N}(\mathbf{v}) & =\sum_{L=1}^{N} \sum_{m=-L}^{L} \widehat{\mathbf{v}}_{L, m, M} \mathbf{Z}_{L, m}, \\
\widehat{\mathbf{v}}_{L, m, M} & =Q_{M}\left(\mathbf{v} \cdot \overline{\mathbf{Z}_{L, m}}\right)=\left(\mathbf{v}, \mathbf{Z}_{L, m}\right)_{M} .
\end{aligned}
$$

With $M$ chosen to satisfy (3.8), it is easy to see that

$$
\mathbf{L}_{N}(\mathbf{v})=\boldsymbol{\Pi}_{N}(\mathbf{v})=\mathbf{v}, \quad \mathbf{v} \in V_{N},
$$

and for $\mathbf{v} \in H \cap C(T S)$ and $\mathbf{w} \in H \cap C^{k}(T S)$,

$$
\left\|\mathbf{L}_{N}(\mathbf{v})\right\| \leq C\|\mathbf{v}\|_{\infty}, \quad \quad\left\|\mathbf{w}-\mathbf{L}_{N}(\mathbf{w})\right\| \leq C N^{-k}\|\mathbf{w}\|_{C^{k}(T S)}
$$

where the last two inequalities follow from simple arguments used in Theorem 13 and Lemma 14 of [24]. In particular, since $\mathcal{D}\left(\mathbf{A}^{s+1 / 2}\right) \subset H \cap C^{2 s}(T S)$, for an integer $2 s$, using (3.1),

$$
\left\|\mathbf{f}-\mathbf{L}_{N}(\mathbf{f})\right\| \leq C N^{-2 s}\|\mathbf{f}\|_{C^{2 s}(T S)}, \quad t \in[0, T] .
$$

Next we consider the Stokes projection in $V_{N}$ of the exact unique regular solution $\mathbf{u}(t):=\mathbf{u}(., t)$ of (2.1). For each fixed $t$, the Stokes projection $\widetilde{\mathbf{u}}_{N} \in V_{N}$ of $\mathbf{u}$ is defined by

$$
\left(\mathbf{A} \widetilde{\mathbf{u}}_{N}, \mathbf{v}\right)=(\mathbf{A u}, \mathbf{v}), \quad \mathbf{v} \in V_{N} .
$$

Since $\boldsymbol{\Pi}_{N} \mathbf{A}=\mathbf{A} \boldsymbol{\Pi}_{N}$, it follows that $\widetilde{\mathbf{u}}_{N}=\boldsymbol{\Pi}_{N}(\mathbf{u})$. Following standard techniques in finite element analysis, the Stokes projection of $\mathbf{u}$ plays an important role as a comparison function in the main analysis in the next section.

Theorem 3.1. Let $\mathbf{u}_{0}$ and $\mathbf{f}$ satisfy (3.1). Then

$$
\left\|\mathbf{u}-\widetilde{\mathbf{u}}_{N}\right\| \leq C \lambda_{N+1}^{-s-1 / 2} e^{-\sigma(t) \lambda_{N+1}^{1 / 2}} \leq C N^{-2 s-1} e^{-\sigma(t) N}, \quad t \in[0, T],
$$

where $\sigma(t)$ is as in Theorem 2.1 .

Proof. Using the fact that $\widetilde{\mathbf{u}}_{N}=\boldsymbol{\Pi}_{N} \mathbf{u}$, we have

$$
\begin{aligned}
\left\|\mathbf{u}-\widetilde{\mathbf{u}}_{N}\right\|^{2} & =\sum_{L>N} \sum_{|m| \leq L}\left|\widehat{\mathbf{u}}_{L, m}\right|^{2} \\
& \leq \lambda_{N+1}^{-2 s-1} e^{-2 \sigma(t) \lambda_{N+1}^{1 / 2}} \sum_{L>N} \sum_{|m| \leq L} \lambda_{L}^{2 s+1} e^{2 \sigma(t) \lambda_{L}^{1 / 2}\left|\widehat{\mathbf{u}}_{L, m}\right|^{2}} \\
& \leq \lambda_{N+1}^{-2 s-1} e^{-2 \sigma(t) \lambda_{N+1}^{1 / 2}\left\|\mathbf{A}^{s+1 / 2} e^{\sigma(t) \mathbf{A}^{1 / 2}} \mathbf{u}(t)\right\|^{2}} \\
& \leq C \lambda_{N+1}^{-2 s-1} e^{-2 \sigma(t) \lambda_{N+1}^{1 / 2}}
\end{aligned}
$$

where in the last step we used (2.35). The last inequality in (3.14) follows from the fact that $N^{2} \leq \lambda_{N+1}=(N+1)(N+2)$. 
Theorem 3.2. Let $\mathbf{u}_{0}$, f satisfy (3.1). We assume further that $\mathbf{f}$ is analytic in $\mathbb{T}$ and (2.37) holds. Then for $t \in(0, T)$,

$$
\left\|\frac{d}{d t}\left(\mathbf{u}-\widetilde{\mathbf{u}}_{N}\right)\right\| \leq C \lambda_{N+1}^{-s-1 / 2} e^{-\psi_{1}(t) \lambda_{N+1}^{1 / 2}} \leq C N^{-2 s-1} e^{-\psi_{1}(t) N},
$$

where $\psi_{1}(t)=\min \left\{(1-1 / \sqrt{2}) t, T^{* *}, \sigma_{1}\right\}$, and $T^{* *}$ is as in Theorem 2.3 .

Proof. Let $t \in(0, T)$ be fixed. Let $\mathbf{p}_{N}(\zeta)=\left(\mathbf{u}-\widetilde{\mathbf{u}}_{N}\right)(\zeta)$ be the standard complexification of $\mathbf{u}-\widetilde{\mathbf{u}}_{N}$ at $\zeta=r e^{i \theta}$. Using Theorem 2.3 and the Cauchy integral formula,

$$
\frac{d \mathbf{p}_{N}(t)}{d t}=\frac{1}{2 \pi i} \int_{\Gamma} \frac{\mathbf{p}_{N}(\zeta)}{(t-\zeta)^{2}} d \zeta
$$

where for $t>0, \Gamma$ is a circle in the $\zeta$ plane with center $(t, 0)$ and radius $\min \left\{t / \sqrt{2}, T^{* *} / \sqrt{2}, T-t\right\}$, a condition that ensures that $\zeta=r e^{i \theta} \in \Gamma$ lies in the region $\mathbb{T}$ with $|\operatorname{Im} \zeta| \leq T^{* *} / \sqrt{2}$. Using the fact that $\widetilde{\mathbf{u}}_{N}=\boldsymbol{\Pi}_{N} \mathbf{u}$, for $\zeta=r e^{i \theta} \in \Gamma$ we have, from Theorem 2.3 ,

$$
\begin{aligned}
& \left\|\mathbf{p}_{N}(\zeta)\right\|=\left\|\mathbf{u}(\zeta)-\widetilde{\mathbf{u}}_{N}(\zeta)\right\|^{2} \\
& =\sum_{L>N} \sum_{|m| \leq L}\left|\widehat{\mathbf{u}}_{L, m}\right|^{2} \\
& \leq \lambda_{N+1}^{-2 s-1} e^{-2 \psi(r \cos \theta) \lambda_{N+1}^{1 / 2}} \sum_{L>N} \sum_{|m| \leq L} \lambda_{L}^{2 s+1} e^{2 \psi(r \cos \theta) \lambda_{L}^{1 / 2}}\left|\widehat{\mathbf{u}}_{L, m}\right|^{2} \\
& \leq \lambda_{N+1}^{-2 s-1} e^{-2 \psi(r \cos \theta) \lambda_{N+1}^{1 / 2}}\left\|\mathbf{A}^{s+1 / 2} e^{\psi(r \cos \theta) \mathbf{A}^{1 / 2}} \mathbf{u}(\zeta)\right\|^{2} \\
& \leq C \lambda_{N+1}^{-2 s-1} e^{-2 \psi(r \cos \theta) \lambda_{N+1}^{1 / 2}} \\
& \leq C N^{-2(2 s+1)} e^{-2 \psi(r \cos \theta) N} .
\end{aligned}
$$

For $\zeta=r e^{i \theta} \in \Gamma$ it is easily seen that $r \cos \theta \geq(1-1 / \sqrt{2}) t$, and hence that

$$
\psi(r \cos \theta) \geq \min \left\{(1-1 / \sqrt{2}) t, T^{* *}, \sigma_{1}\right\}=: \psi_{1}(t) .
$$

By using (3.16) in (3.14) we get

$$
\left\|\frac{d}{d t}\left(\mathbf{u}-\widetilde{\mathbf{u}}_{N}\right)(t)\right\| \leq \frac{1}{2 \pi} \int_{\Gamma} \frac{\left\|\mathbf{p}_{N}(\zeta)\right\|}{|t-\zeta|^{2}} d \zeta \leq C N^{-2 s-1} e^{-\psi_{1}(t) N} .
$$

\section{A pseudospectral quadrature method}

We are now ready to describe, analyze, and implement a spectrally accurate scheme to compute approximate solutions of the NSE (2.1) in $V_{N}$, through its weak formulation (2.27). The task is then to compute $\mathbf{u}_{N}(\cdot, t) \in V_{N}$ for $t \in[0, T]$ with $\mathbf{u}_{N}(0, \widehat{\mathbf{x}})=\mathbf{L}_{N} \mathbf{u}_{0}(\widehat{\mathbf{x}}), \widehat{\mathbf{x}} \in S$, satisfying the (spatially) discrete system of ordinary differential equations

$$
\begin{aligned}
& \frac{d}{d t}\left(\mathbf{u}_{N}, \mathbf{v}\right)_{M}+b\left(\mathbf{u}_{N}, \mathbf{u}_{N}, \mathbf{v}\right)_{M}+\nu\left(\operatorname{Curl}_{\widehat{\mathbf{x}}} \mathbf{u}_{N}, \operatorname{Curl}_{\widehat{\mathbf{x}}} \mathbf{v}\right)_{M} \\
& +\left(\mathbf{C u}_{N}, \mathbf{v}\right)_{M}=(\mathbf{f}, \mathbf{v})_{M},
\end{aligned}
$$

for all $\mathbf{v} \in V_{N}$ and prove that the scheme is spectrally accurate with respect to the parameter $N$ (that is, converges with rate determined by the smoothness of the given data), and demonstrate the theory with numerical experiments. 
Using (3.2), the exactness properties of the discrete inner product, (3.8), (2.10), (2.16), (2.23), and (2.29), the system (4.1) can be written as

$$
\begin{gathered}
\left(\frac{d}{d t} \mathbf{u}_{N}+\nu \mathbf{A} \mathbf{u}_{N}+\mathbf{B}\left(\mathbf{u}_{N}, \mathbf{u}_{N}\right)+\mathbf{C} \mathbf{u}_{N}, \mathbf{Z}_{L, m}\right)=\left(\mathbf{f}, \mathbf{Z}_{L, m}\right)_{M}, \\
L=1, \ldots, N ; m=-L, \ldots, L
\end{gathered}
$$

4.1. Stability and convergence analysis. First we establish the stability of the approximate solution $\mathbf{u}_{N}$ of (4.24). That is, similar to [6, Proposition 9.1], we prove that $\max _{t \in[0, T]}\left\|\mathbf{u}_{N}\right\|_{V}$ is uniformly bounded with the bound depending only on the initial data, forcing function, and the viscosity term in (4.24).

Theorem 4.1. Let $\mathbf{u}_{0}$ and $\mathbf{f}$ satisfy (3.1). Let $N \geq 1$ be an integer. Let $\mathbf{u}_{N}$ be the solution of the pseudospectral quadrature Galerkin system (4.1). Then there exists a constant $C$ depending on $\nu,\left\|\mathbf{u}_{0}\right\|_{V}$ and $\|\mathbf{f}\|_{\infty}:=\max _{t \in[0, T]}\|\mathbf{f}(t)\|_{C(T S)}$ so that

$$
\max _{t \in[0, T]}\left\|\mathbf{u}_{N}\right\|_{V} \leq C .
$$

Proof. The proof follows by repeating the arguments described in the first four pages of [6. Section 9] (proving [6. Proposition 9.1]), provided that we establish [6, inequalities (9.3) and (9.13)] for our system (4.1) on the spherical surface with additional Coriolis term and quadrature approximations.

Using (2.26), (2.29) and the exactness of the quadrature rule (given by (3.7) and (3.8)), we have $\left(\mathbf{B}\left(\mathbf{u}_{N}, \mathbf{u}_{N}\right), \mathbf{u}_{N}\right)_{M}=0$. Using (4.27), the symmetry of the coefficients of $\mathbf{u}_{N}$ in (4.24) and the exactness of the quadrature, we get

$$
\left(\mathbf{C} \mathbf{u}_{N}, \mathbf{u}_{N}\right)_{M}=\left(\mathbf{C} \mathbf{u}_{N}, \mathbf{u}_{N}\right)=(-2 \Omega i) \sum_{L=1}^{N} \lambda_{L}^{-1} \sum_{|m| \leq L} m\left|\alpha_{L, m}\right|^{2}=0 .
$$

By taking $\mathbf{v}$ to be $\mathbf{u}_{N}$ in (4.1) and using $\left\|\mathbf{u}_{N}\right\|^{2}=\left(\mathbf{u}_{N}, \mathbf{u}_{N}\right)_{M},\left\|\mathbf{u}_{N}\right\|_{V}^{2}=\left(\mathbf{A} \mathbf{u}_{N}, \mathbf{u}_{N}\right)_{M}$, (4.3), Young's inequality, and the fact that all the eigenvalues $\lambda_{J}$ of $\mathbf{A}$ (corresponding to eigenvectors in $\mathbf{u}_{N}$ ) satisfy $\lambda_{J} \geq \lambda_{1}=2, J=1, \ldots, N$, we obtain

$$
\begin{gathered}
\frac{1}{2} \frac{d}{d t}\left\|\mathbf{u}_{N}\right\|^{2}+\nu\left\|\mathbf{u}_{N}\right\|_{V}^{2}=\left(\mathbf{f}, \mathbf{u}_{N}\right)_{M} \leq\|\mathbf{f}\|_{\infty}\left\|\mathbf{u}_{N}\right\| \\
\leq \frac{\|\mathbf{f}\|_{\infty}^{2}}{4 \nu}+\nu\left\|\mathbf{u}_{N}\right\|^{2} \leq \frac{\|\mathbf{f}\|_{\infty}^{2}}{4 \nu}+\frac{\nu}{2}\left\|\mathbf{u}_{N}\right\|_{V}^{2}
\end{gathered}
$$

Hence, for our discrete system (4.1), we obtain [6, Inequality (9.3)]:

$$
\frac{d}{d t}\left\|\mathbf{u}_{N}\right\|^{2}+\nu\left\|\mathbf{u}_{N}\right\|_{V}^{2} \leq \frac{\|\mathbf{f}\|_{\infty}^{2}}{\nu \lambda_{1}}
$$

Again, using (4.27), the exactness of the quadrature rule, eigenfunction properties of $\mathbf{A}$, and the symmetry of the coefficients of $\mathbf{u}_{N}$ in (4.24), we get

$$
\left(\mathbf{C u}_{N}, \mathbf{A} \mathbf{u}_{N}\right)=(-2 \Omega i) \sum_{L=1}^{N} \sum_{|m| \leq L} m\left|\alpha_{L, m}\right|^{2}=0
$$

By taking $\mathbf{v}$ to be $\mathbf{A} \mathbf{u}_{N}$ in equation (4.1), and using $\left\|\mathbf{A} \mathbf{u}_{N}\right\|^{2}=\left(\mathbf{A} \mathbf{u}_{N}, \mathbf{A} \mathbf{u}_{N}\right)_{M}$, (3.8), and (4.3), we obtain

$$
\frac{1}{2} \frac{d}{d t}\left\|\mathbf{u}_{N}\right\|_{V}^{2}+\nu\left\|\mathbf{A} \mathbf{u}_{N}\right\|^{2}+b\left(\mathbf{u}_{N}, \mathbf{u}_{N}, \mathbf{A} \mathbf{u}_{N}\right)=\left(\mathbf{f}, \mathbf{A} \mathbf{u}_{N}\right)_{M} .
$$


Using [16. Lemma 3.1], $b\left(\mathbf{u}_{N}, \mathbf{u}_{N}, \mathbf{A} \mathbf{u}_{N}\right)=0$. The term $\left(\mathbf{f}, \mathbf{A} \mathbf{u}_{N}\right)_{M}$ can be estimated by using the exactness of the quadrature and Young's inequality:

$$
\left(\mathbf{f}, \mathbf{A} \mathbf{u}_{N}\right)_{M} \leq\|\mathbf{f}\|_{\infty}\left\|\mathbf{A} \mathbf{u}_{N}\right\| \leq \frac{\|\mathbf{f}\|_{\infty}^{2}}{2 \nu}+\frac{\nu}{2}\left\|\mathbf{A} \mathbf{u}_{N}\right\|^{2} .
$$

Hence, we obtain a stronger version of [6, Inequality (9.13)] for our quadrature discrete scheme (4.1):

$$
\frac{d}{d t}\left\|\mathbf{u}_{N}\right\|_{V}^{2}+\nu\left\|\mathbf{A} \mathbf{u}_{N}\right\|^{2} \leq \frac{\|\mathbf{f}\|_{\infty}^{2}}{\nu \lambda_{1}} .
$$

Thus, the result follows from arguments in [6, Pages 74-77].

Next, using Theorems 3.1, 3.2, and 4.1 we prove the spectral convergence of the solution $\mathbf{u}_{N}$ of (4.2) to the solution of $\mathbf{u}$ of (2.27).

Theorem 4.2. Let $\mathbf{u}_{0}$, $\mathbf{f}$ satisfy (2.37) and (3.1). Then there exists a $T^{\#}>0$, depending only on $\nu, \mathbf{f}, \mathbf{u}_{0}$ and the uniform bound in (2.45) (and hence there exists $\left.0<\mu(t)<\min \left\{t, T^{\#}, \sigma_{1}\right\}\right)$ such that for all $t \in(0, T)$,

$$
\left\|\mathbf{u}-\mathbf{u}_{N}\right\| \leq C\left[N^{-2 s-1} e^{-\mu(t) N}+\left\|\mathbf{\Pi}_{N} \mathbf{f}-\mathbf{L}_{N} \mathbf{f}\right\|\right] .
$$

In particular, with $2 s$ being an integer,

$$
\left\|\mathbf{u}-\mathbf{u}_{N}\right\| \leq C N^{-2 s} .
$$

Proof. Let $\mathbf{w}_{N}=\widetilde{\mathbf{u}}_{N}-\mathbf{u}_{N}$, where the comparison function $\widetilde{\mathbf{u}}_{N}$ is the solution of (3.13). Since $\mathbf{u}-\mathbf{u}_{N}=\mathbf{p}_{N}+\mathbf{w}_{N}$, where $\mathbf{p}_{N}=\mathbf{u}-\widetilde{\mathbf{u}}_{N}$, in view of Theorems 3.1 and 3.2. existence of $T^{\#}$ and $\mu(t)$ follows and it is sufficient to show that $\left\|\mathbf{w}_{N}\right\| \leq$ $C\left[N^{-2 s-1} e^{-\mu(t) N}+\left\|\mathbf{\Pi}_{N} \mathbf{f}-\mathbf{L}_{N} \mathbf{f}\right\|\right]$. For any $\mathbf{v} \in V_{N}$, using (2.28), (3.13), and (4.2),

$$
\begin{aligned}
& \left(\left(\mathbf{w}_{N}\right)_{t}, \mathbf{v}\right)+\nu\left(\mathbf{A} \mathbf{w}_{N}, \mathbf{v}\right)+\left(\mathbf{C} \mathbf{w}_{N}, \mathbf{v}\right) \\
& =\left(\left(\widetilde{\mathbf{u}}_{N}\right)_{t}, \mathbf{v}\right)+\nu\left(\mathbf{A} \widetilde{\mathbf{u}}_{N}, \mathbf{v}\right)+\left(\mathbf{C} \widetilde{\mathbf{u}}_{N}, \mathbf{v}\right)-\left(\left(\mathbf{u}_{N}\right)_{t}, \mathbf{v}\right)-\nu\left(\mathbf{A} \mathbf{u}_{N}, \mathbf{v}\right)-\left(\mathbf{C} \mathbf{u}_{N}, \mathbf{v}\right) \\
& =\left(\left(\widetilde{\mathbf{u}}_{N}\right)_{t}, \mathbf{v}\right)+\nu\left(\mathbf{A} \widetilde{\mathbf{u}}_{N}, \mathbf{v}\right)+\left(\mathbf{C} \widetilde{\mathbf{u}}_{N}, \mathbf{v}\right)-(\mathbf{f}, \mathbf{v})_{M}+\left(\mathbf{B}\left(\mathbf{u}_{N}, \mathbf{u}_{N}\right), \mathbf{v}\right) \\
& =\left(\left(\widetilde{\mathbf{u}}_{N}\right)_{t}, \mathbf{v}\right)+\nu(\mathbf{A u}, \mathbf{v})+\left(\mathbf{C} \widetilde{\mathbf{u}}_{N}, \mathbf{v}\right)-(\mathbf{f}, \mathbf{v})_{M}+\left(\mathbf{B}\left(\mathbf{u}_{N}, \mathbf{u}_{N}\right), \mathbf{v}\right) \\
& =\left(\left(\widetilde{\mathbf{u}}_{N}-\mathbf{u}\right)_{t}, \mathbf{v}\right)+(\mathbf{f}, \mathbf{v})-(\mathbf{f}, \mathbf{v})_{M}+\left(\mathbf{C} \widetilde{\mathbf{u}}_{N}-\mathbf{C u}, \mathbf{v}\right)+\left(\mathbf{B}\left(\mathbf{u}_{N}, \mathbf{u}_{N}\right)-\mathbf{B}(\mathbf{u}, \mathbf{u}), \mathbf{v}\right) .
\end{aligned}
$$

Using the orthogonal projection $\boldsymbol{\Pi}_{N}$ in (3.2), we can write this relation in functional form as

$$
\begin{aligned}
\frac{d \mathbf{w}_{N}}{d t}=( & -\nu \mathbf{A}-\mathbf{C}) \mathbf{w}_{N}-\boldsymbol{\Pi}_{\mathbf{N}}\left[\left(\mathbf{p}_{N}\right)_{t}+\mathbf{C p}_{N}\right] \\
& +\boldsymbol{\Pi}_{N} \mathbf{f}-\mathbf{L}_{N} \mathbf{f}+\boldsymbol{\Pi}_{N}\left[\mathbf{B}\left(\mathbf{u}_{N}, \mathbf{u}_{N}\right)-\mathbf{B}(\mathbf{u}, \mathbf{u})\right] .
\end{aligned}
$$

Integrating with respect to $t$ and using $\mathbf{w}_{N}(0)=0$, we have

$$
\begin{aligned}
\mathbf{w}_{N}(t)= & \int_{0}^{t} e^{-(t-s)(\nu \mathbf{A}+\mathbf{C})}\left[-\boldsymbol{\Pi}_{N}\left(\frac{d}{d s} \mathbf{p}_{N}+\mathbf{C} \mathbf{p}_{N}\right)+\boldsymbol{\Pi}_{N} \mathbf{f}-\mathbf{L}_{N} \mathbf{f}\right](s) d s \\
& +\int_{0}^{t} e^{-(t-s)(\nu \mathbf{A}+\mathbf{C})} \boldsymbol{\Pi}_{N}\left[\mathbf{B}\left(\mathbf{u}_{N}, \mathbf{u}_{N}\right)-\mathbf{B}(\mathbf{u}, \mathbf{u})\right](s) d s .
\end{aligned}
$$

Let

$$
R_{N}(\epsilon, t-s)=\left\|\nu^{\epsilon} \boldsymbol{\Pi}_{N} \mathbf{A}^{\epsilon} e^{-(t-s)(\nu \mathbf{A}+\mathbf{C})}\right\| .
$$


By using (2.31), with $\delta \in(1 / 2,1)$, and the uniform boundedness of the orthogonal projection $\boldsymbol{\Pi}_{N}$, we get

$$
\begin{aligned}
& \left\|e^{-(t-s)(\nu \mathbf{A}+\mathbf{C})} \boldsymbol{\Pi}_{N}\left[\mathbf{B}\left(\mathbf{u}_{N}, \mathbf{u}_{N}\right)-\mathbf{B}(\mathbf{u}, \mathbf{u})\right]\right\| \\
& \quad \leq \frac{R_{N}(\delta, t-s)}{\nu^{\delta}}\left\|\mathbf{A}^{-\delta} \boldsymbol{\Pi}_{N}\left(\mathbf{B}\left(\mathbf{u}_{N}, \mathbf{u}_{N}\right)-\mathbf{B}(\mathbf{u}, \mathbf{u})\right)\right\| \\
& \quad \leq C R_{N}(\delta, t-s)\left\|\mathbf{u}_{N}-\mathbf{u}\right\| .
\end{aligned}
$$

Taking norms and using $\left\|\mathbf{u}_{N}-\mathbf{u}\right\| \leq\left\|\mathbf{w}_{N}\right\|+\left\|\mathbf{p}_{N}\right\|$ together with (4.10) and (4.11) in (4.9), we obtain

$$
\begin{aligned}
\left\|\mathbf{w}_{N}(t)\right\| \leq & {\left[\left\|\frac{d}{d t} \mathbf{p}_{N}\right\|+\left\|\mathbf{C} \mathbf{p}_{N}\right\|+\left\|\mathbf{\Pi}_{N} \mathbf{f}-\mathbf{L}_{N} \mathbf{f}\right\|\right] \int_{0}^{t} R_{N}(0, t-s) d s } \\
& +C \int_{0}^{t} R_{N}(\delta, t-s)\left(\left\|\mathbf{p}_{N}(s)\right\|+\left\|\mathbf{w}_{N}(s)\right\|\right) d s .
\end{aligned}
$$

Using Gronwall's inequality, we obtain for each $t \in[0, T]$,

$$
\begin{gathered}
\left\|\mathbf{w}_{N}(t)\right\| \leq C\left\{\left[\left\|\frac{d}{d t} \mathbf{p}_{N}\right\|+\left\|\mathbf{C p}_{N}\right\|+\left\|\mathbf{\Pi}_{N} \mathbf{f}-\mathbf{L}_{N} \mathbf{f}\right\|\right] \int_{0}^{t} R_{N}(0, t-s) d s\right. \\
\left.+\left\|\mathbf{p}_{N}\right\| \int_{0}^{t} R_{N}(\delta, t-s) d s\right\} .
\end{gathered}
$$

For $\epsilon \in[0,1]$, we have to bound

$$
\int_{0}^{t} R_{N}(\epsilon, t-s) d s=\int_{0}^{t} R_{N}(\epsilon, r) d r
$$

Using also (4.10) and (4.27),

$$
R_{N}(\epsilon, r) \leq \max _{1 \leq L \leq N ;|m| \leq L}\left|\left(\nu \lambda_{L}\right)^{\epsilon} e^{-\nu \lambda_{L} r} e^{-2 \Omega i r m \lambda_{L}^{-1 / 2}}\right| \leq \max _{z \in\left[\nu \lambda_{1}, \nu \lambda_{N}\right]} z^{\epsilon} e^{-r z}
$$

Thus,

$$
R_{N}(r) \leq \begin{cases}\left(\nu \lambda_{N}\right)^{\epsilon} e^{-\nu \lambda_{N} r} & \text { if } r \leq \epsilon /\left(\nu \lambda_{N}\right) \\ \epsilon^{\epsilon} e^{-\epsilon} r^{-\epsilon} & \text { if } \epsilon /\left(\nu \lambda_{N}\right) \leq r \leq \epsilon /\left(\nu \lambda_{1}\right) \\ \left(\nu \lambda_{1}\right)^{\epsilon} e^{-\nu \lambda_{1} r} & \text { if } r \geq \epsilon /\left(\nu \lambda_{1}\right)\end{cases}
$$

With

$I_{1}=\left[0, \epsilon /\left(\nu \lambda_{N}\right)\right] \cap[0, t], \quad I_{2}=\left[\epsilon /\left(\nu \lambda_{N}\right), \epsilon /\left(\nu \lambda_{1}\right)\right] \cap[0, t], \quad I_{3}=\left[\epsilon /\left(\nu \lambda_{1}\right), t\right] \cap[0, t]$, the interval of integration in (4.13) can be subdivided into these three intervals. In particular, using (4.14) and (4.15), we get

$$
\begin{gathered}
\int_{I_{1}} R_{N}(r) d r \leq \int_{I_{1}}\left(\nu \lambda_{N}\right)^{\epsilon} e^{-\nu \lambda_{N} r} d r \leq \frac{\left(1-e^{-\epsilon}\right)}{\left(\nu \lambda_{N}\right)^{1-\epsilon}} \leq C, \\
\int_{I_{2}} R_{N}(r) d r \leq \int_{I_{2}} \epsilon^{\epsilon} e^{-\epsilon} r^{-\epsilon} d r \\
\leq \frac{\epsilon e^{-\epsilon}}{(1-\epsilon)}\left[\left(\frac{1}{\nu \lambda_{1}}\right)^{(1-\epsilon)}-\left(\frac{1}{\nu \lambda_{N}}\right)^{(1-\epsilon)}\right] \leq C,
\end{gathered}
$$


and

$$
\int_{I_{3}} R_{N}(r) d r \leq \int_{I_{3}}\left(\nu \lambda_{1}\right)^{\epsilon} e^{\nu \lambda_{1} r} d r \leq \frac{\left(e^{-\epsilon}-e^{-\nu \lambda_{1} t}\right)}{\left(\nu \lambda_{1}\right)^{1-\epsilon}} \leq C .
$$

Using (4.16)-(4.18) in (4.13), we get

$$
\int_{0}^{t} R_{N}(\epsilon, t-s) d s \leq C, \quad \epsilon \in[0,1] .
$$

Substituting this in (4.12), we get

$$
\left\|\mathbf{w}_{N}(t)\right\| \leq C\left[\left\|\frac{d}{d t} \mathbf{p}_{N}\right\|+\left\|\mathbf{C p}_{N}\right\|+\left\|\mathbf{p}_{N}\right\|+\left\|\boldsymbol{\Pi}_{N} \mathbf{f}-\mathbf{L}_{N} \mathbf{f}\right\|\right] .
$$

The term $\left\|\mathbf{C} \mathbf{p}_{N}\right\|$ in (4.20) can be simplified using (2.23) and the fact that $\mathbf{p}_{N}$ is tangential (and hence $\widehat{\mathbf{x}} \cdot \mathbf{p}_{N}(\widehat{\mathbf{x}})=0$ ),

$$
\left[\widehat{\mathbf{x}} \times \mathbf{p}_{N}(\widehat{\mathbf{x}})\right] \cdot\left[\overline{\left(\widehat{\mathbf{x}} \times \mathbf{p}_{N}(\widehat{\mathbf{x}})\right.}\right]=[\widehat{\mathbf{x}} \cdot \widehat{\mathbf{x}}]\left[\mathbf{p}_{N}(\widehat{\mathbf{x}}) \cdot \overline{\mathbf{p}_{N}(\widehat{\mathbf{x}})}\right]=\left\|\mathbf{p}_{N}\right\|^{2},
$$

and hence

$$
\left\|\mathbf{w}_{N}(t)\right\| \leq C\left[\left\|\frac{d}{d t} \mathbf{p}_{N}\right\|+\left\|\mathbf{p}_{N}\right\|+\left\|\boldsymbol{\Pi}_{N} \mathbf{f}-\mathbf{L}_{N} \mathbf{f}\right\|\right] .
$$

Hence, from Theorems 3.1 and 3.2 , for all $t \in(0, T)$ we have

$$
\left\|\mathbf{u}-\mathbf{u}_{N}\right\| \leq C\left[N^{-2 s-1} e^{-\mu(t) N}+\left\|\mathbf{\Pi}_{N} \mathbf{f}-\mathbf{L}_{N} \mathbf{f}\right\|\right] .
$$

In particular, using (3.5) and (3.12) with $2 s$ being an integer, we get

$$
\left\|\boldsymbol{\Pi}_{N} \mathbf{f}-\mathbf{L}_{N} \mathbf{f}\right\| \leq\left\|\boldsymbol{\Pi}_{N} \mathbf{f}-\mathbf{f}\right\|+\left\|\mathbf{f}-\mathbf{L}_{N} \mathbf{f}\right\| \leq C N^{-2 s} .
$$

Now the result (4.8) follows from (4.22) and (4.23).

4.2. Adaptive and fast implementation of the pseudospectral method. Having established spectrally accurate convergence of the spatially discrete scheme (4.2), for implementation of the scheme (4.2) to simulate stable and accurate solutions of (2.1) and compare with benchmark random flow simulations in the literature, we need to discretize the time derivative operator $\frac{d}{d t}$ in (4.2). Further, at each discrete time step we develop a (FFT-based) fast evaluation technique to set up the resulting fully discrete nonlinear system with spatial $\mathcal{O}\left(N^{4}\right)$ complexity. First we consider discretization of $\frac{d}{d t}$ in (4.2).

In order to the make (4.2) fully discrete in space and time, and hence compute the $N^{2}+2 N$ unknown time-dependent coefficients in the representation of the tangential divergence-free approximate real velocity vector fields

$$
\begin{gathered}
\mathbf{u}_{N}(\widehat{\mathbf{x}}, t):=\sum_{L=1}^{N} \sum_{|m| \leq L} \alpha_{L, m}(t) \mathbf{Z}_{L, m}(\widehat{\mathbf{x}}), \quad \alpha_{L, m}=\bar{\alpha}_{L,-m}, \\
\alpha_{L, m}(0)=\left(\mathbf{u}_{0}, \mathbf{Z}_{L, m}\right)_{M}
\end{gathered}
$$

for $\widehat{\mathbf{x}} \in S$ and $t \geq 0$, the standard fixed-time-step backward-Euler (or CrankNicolson) Galerkin approach could be used in (4.1), leading to a first-order (or a second-order, respectively) in time nonadaptive scheme [13]. However, due to the complicated unknown flow behavior of the NSE solutions, when the initial states are random, it is more efficient instead to integrate (4.1) using a combination of multi-order integration formulas that allow adaptive choice of time step, leading to 
computation of solutions with a specified accuracy in time. In this paper we follow the latter approach.

For implementation purposes, we first need to substitute (4.24) in (4.2). We write the resulting $N^{2}+2 N$-dimensional system of ordinary differential equations (ODE), for the unknown $N^{2}+2 N$ time-dependent coefficients of $\mathbf{u}_{N}(\widehat{\mathbf{x}}, t)$ in (4.24), as

$$
\frac{d}{d t} \boldsymbol{\alpha}(t)=\mathbf{F}(t, \boldsymbol{\alpha}(t))
$$

It is well known that such nonlinear ODE systems are stiff, and hence it is important to use time discretization techniques with a large stability region [14. Further, it is important to use high-order implicit formulas whenever possible, but the high-order discretization formulas are appropriate only at those time steps where the unknown exact solution is smooth.

For practical problems such as the Navier-Stokes equations (with initial random state) where the necessary spatial discretization (at each time step) is expensive, it is important to optimize computing time by simulating only up to a required accuracy by choosing adaptive discrete time steps. Unlike the adaptive spatial mesh for elliptic PDEs based on the a posteriori estimates, adaptive time steps can be computed by comparing numerical solutions obtained using two distinct order formulas 14 and hence simulation using multi-order formulas is appropriate.

In particular, for practical realization of large stiff nonlinear ODE systems, multiorder implicit backward differentiation formulas (BDF) and their generalizations such as the numerical differential formulas (NDF) are most appropriate. The implicit NDF formula of order $p$ (NDFp) with a parameter $\kappa_{p}$ (so that $\kappa_{p}=0$ corresponds to BDFp) for the system (4.25), with $\boldsymbol{\alpha}_{n} \approx \boldsymbol{\alpha}\left(t_{n}\right)$ and $\nabla^{m}$ denoting the $m$-th Newton backward difference operator, is

$$
\sum_{m=1}^{p} \frac{1}{m} \nabla^{m} \boldsymbol{\alpha}_{n+1}=h \mathbf{F}\left(t_{n+1}, \boldsymbol{\alpha}_{n+1}\right)+\kappa_{p} \nabla^{p+1} \boldsymbol{\alpha}_{n+1} \sum_{j=1}^{p} \frac{1}{j} .
$$

It is well known [14 that BDFp (and hence NDFp) are unstable for $p>6$, and for $p=6$ the stability region is small and hence not practically useful in our case. Further, the celebrated Dahlquist barrier [14] implies that BDFp (and hence NDFp) cannot be absolutely stable [that is, $A(\alpha)$-stable with $\alpha=90^{\circ}$ ] for $p>2$.

Following details in [25], for simulation of (4.25) we use multi-order NDFp with $p=1,2,3,4,5$ (and respective $\left.\kappa_{p}=-0.1850,-1 / 9,-0.0823,-0.0415,0\right)$ and these are $A\left(\alpha_{p}\right)$-stable, with respective $\alpha_{p}=90^{\circ}, 90^{\circ}, 80^{\circ}, 66^{\circ}, 51^{\circ}$. For $p=1,2,3,4,5$, NDFp is more accurate than BDFp, however, NDFp has a slightly smaller stability angle compared to BDFp only for $p=3,4$ (with respective $\alpha_{p}=86^{\circ}, 73^{\circ}$ ) and the same stability angle for $p=1,2,5$.

For each fixed time discretization step, the computational cost is dominated by evaluation of $\mathbf{F}\left(t_{n+1}, \boldsymbol{\alpha}_{n+1}\right)$ in (4.26) and it is important to have an efficient method to set up the spatial part of the nonlinear system (4.2). Using the spectral properties of the Stokes operator A given by (2.17), the linear second term in (4.2) is trivial to evaluate using the diagonal matrix consisting of the eigenvalues of $\mathbf{A}$.

The Coriolis term can be evaluated similarly using the identity [5, equation (24)]

$$
\left(\mathbf{C} \operatorname{Curl} Y_{J, k}, \mathbf{Z}_{L, m}\right)=\left(2 \Omega \cos \theta \widehat{\mathbf{x}} \times \operatorname{Curl} Y_{J, k}, \mathbf{Z}_{L, m}\right)=-2 \Omega i \frac{m}{\lambda_{L}^{1 / 2}} \delta_{L, J} \delta_{k, m} .
$$


For the first component in the nonlinear third term in (4.2), we use (2.7) to write

$$
\begin{aligned}
\mathbf{B}\left(\mathbf{Z}_{R, s}, \mathbf{Z}_{J, k}\right) & =-\frac{1}{\sqrt{\lambda_{R} \lambda_{J}}} \mathbf{P}_{\mathbf{C u r l}}\left(\Delta Y_{R, s} \operatorname{Grad} Y_{J, k}\right), \\
\left(\mathbf{B}\left(\mathbf{Z}_{R, s}, \mathbf{Z}_{J, k}\right), \mathbf{Z}_{L, m}\right) & =\sqrt{\frac{\lambda_{R}}{\lambda_{J} \lambda_{L}}}\left(Y_{R, s} \mathbf{G r a d} Y_{J, k}, \widehat{\mathbf{x}} \times \operatorname{Grad} Y_{L, m}\right) .
\end{aligned}
$$

It is convenient to write $\operatorname{Grad} Y_{J, k}$ and $\widehat{\mathbf{x}} \times \operatorname{Grad} Y_{L, m}$ in terms of expressions similar to those in (2.18). Such explicit representations are also useful for the efficient evaluation of the $N^{2}$ Fourier coefficients $\left(\mathbf{f}, \mathbf{Z}_{L, m}\right)_{M}$ of the source term in (4.2), and eventually for the computation of the vorticity field.

In order to express the tangential (and normal, needed for computing the approximate vorticity from $\mathbf{u}_{N}$ ) vector harmonics as a linear combination of the scalar harmonics (2.18), we first recall, from the classical quantum mechanics literature (see, for example, [30]), the covariant spherical basis vectors

$$
\begin{gathered}
\mathbf{e}_{+1}=-\frac{1}{\sqrt{2}}\left([1,0,0]^{T}+i[0,1,0]^{T}\right), \quad \mathbf{e}_{0}=[0,0,1]^{T}, \\
\mathbf{e}_{-1}=\frac{1}{\sqrt{2}}\left([1,0,0]^{T}-i[0,1,0]^{T}\right),
\end{gathered}
$$

and the Clebsch-Gordan coefficients,

$$
C_{j_{1}, m_{1}, j_{2}, m_{2}}^{j, m}:=(-1)^{\left(m+j_{1}-j_{2}\right)} \sqrt{2 j+1}\left(\begin{array}{lll}
j_{1} & j_{2} & j \\
m_{1} & m_{2} & -m
\end{array}\right),
$$

where $\left(\begin{array}{lll}a & b & c \\ \alpha & \beta & \gamma\end{array}\right)$ are the Wigner $3 \mathrm{j}$-symbols given, for example, by the Racah formula,

$$
\begin{aligned}
& \left(\begin{array}{ccc}
a & b & c \\
\alpha & \beta & \gamma
\end{array}\right) \\
& =(-1)^{(a-b-\gamma)} \sqrt{T(a b c)} \sqrt{(a+\alpha) !(a-\alpha) !(b+\beta) !(b-\beta) !(c+\gamma) !(c-\gamma) !} \\
& \quad \times \sum_{t} \frac{(-1)^{t}}{t !(c-b+t+\alpha) !(c-a+t-\beta) !(a+b-c-t) !(a-t-\alpha) !(b-t+\beta) !},
\end{aligned}
$$

where the sum is over all integers $t$ for which the factorials in the denominator all have nonnegative arguments. In particular, the number of terms in the sum is $1+\min \{a \pm \alpha, b \pm \beta, c \pm \gamma, a+b-c, b+c-a, c+a-b\}$. The triangle coefficient $T(a b c)$ is defined by

$$
T(a b c)=\left[\frac{(a+b-c) !(a-b+c) !(-a+b+c) !}{(a+b+c+1) !}\right] .
$$

Below, we require $C_{j_{1}, m_{1}, j_{2}, m_{2}}^{j, m}$ only for some $j_{2}, m_{2} \in\{-1,0,1\}$, and using various symmetry and other known properties (such as $C_{j_{1}, m_{1}, j_{2}, m_{2}}^{j, m}=0$ unless the conditions $\left|j_{1}-j_{2}\right| \leq j \leq j_{1}+j_{2}$ and $m_{1}+m_{2}=m$ hold) of Wigner $3 \mathrm{j}$-symbols, these coefficients can be efficiently pre-computed and stored.

In our computation, we used the following basis functions for the tangential vector fields: (i) $\operatorname{Grad} Y_{L, m},(i i) \widehat{\mathbf{x}} \times \operatorname{Grad} Y_{J, k}$. For the vorticity components of $\mathbf{u}_{N}$, in addition we used: (iii) $\operatorname{Vort} \mathbf{Z}_{J, m}=\operatorname{Curl}_{\widehat{\mathbf{x}}} \times \mathbf{Z}_{J, m}=\lambda_{J}^{1 / 2} \widehat{\mathbf{x}} Y_{J, m}=$ $-\lambda_{J}^{-1 / 2} \widehat{\mathbf{x}} \Delta Y_{J, m}$. In particular, using (4.24) our approximation to the vorticity in (2.2), for a fixed $t \geq 0$ and $\widehat{\mathbf{x}} \in S$, is

$$
\operatorname{Vort} \mathbf{u}_{N}(\widehat{\mathbf{x}}, t)=\operatorname{Curl}_{\widehat{\mathbf{x}}} \mathbf{u}_{N}(\widehat{\mathbf{x}})=\widehat{\mathbf{x}} \Delta \Psi_{N}(\widehat{\mathbf{x}}),
$$


where

$$
\Psi_{N}(\widehat{\mathbf{x}}, t)=-\sum_{L=1}^{N} \sum_{|m| \leq L} \lambda_{L}^{-1 / 2} \alpha_{L, m}(t) Y_{L, m}(\widehat{\mathbf{x}}) .
$$

To facilitate easy application of fast transforms to evaluate these functions at the $M=\mathcal{O}\left(N^{2}\right)$ quadrature points $\left\{\widehat{\boldsymbol{\xi}}_{p, q}=\boldsymbol{p}\left(\theta_{p}, \phi_{q}\right)\right\}$, we represent these three types of fields first as a linear combination of the covariant vectors in (4.30):

$$
\begin{aligned}
\operatorname{Grad} Y_{L, m} & =B_{+1, L, m} \mathbf{e}_{+1}+B_{0, L, m} \mathbf{e}_{0}+B_{-1, L, m} \mathbf{e}_{-1}, \\
\left(\widehat{\mathbf{x}} \times \operatorname{Grad} Y_{J, k}\right) & =D_{+1, J, k} \mathbf{e}_{+1}+D_{0, J, k} \mathbf{e}_{0}+D_{-1, J, k} \mathbf{e}_{-1},
\end{aligned}
$$

With $c_{L}=(L+1) \sqrt{\frac{L}{2 L+1}}, d_{L}=L \sqrt{\frac{L+1}{2 L+1}}$, these coefficients are explicitly given by

$$
\begin{gathered}
B_{+1, L, m}=\left\{c_{L} C_{L-1, m-1,1,1}^{L, m} P_{L-1}^{m-1}(\cos \theta)+d_{L} C_{L+1, m-1,1,1}^{L, m} P_{L+1}^{m-1}(\cos \theta)\right\} e^{i(m-1) \varphi}, \\
B_{0, L, m}=\left\{c_{L} C_{L-1, m, 1,0}^{L, m} P_{L-1}^{m}(\cos \theta)+d_{L} C_{L+1, m, 1,0}^{L, m} P_{L+1}^{m}(\cos \theta)\right\} e^{i m \varphi}, \\
B_{-1, L, m}=\left\{c_{L} C_{L-1, m+1,1,-1}^{L, m} P_{L-1}^{m+1}(\cos \theta)+d_{L} C_{L+1, m+1,1,-1}^{L, m} P_{L+1}^{m+1}(\cos \theta)\right\} e^{i(m+1) \varphi}, \\
D_{+1, J, k}=i \sqrt{\lambda_{J}} C_{J, k-1,1,1}^{J, k} P_{J}^{k-1}(\cos \theta) e^{i(k-1) \varphi} \\
D_{0, J, k}=i \sqrt{\lambda_{J}} C_{J, k, 1,0}^{J, k} P_{J}^{k}(\cos \theta) e^{i k \varphi} \\
D_{-1, J, k}=i \sqrt{\lambda_{J}} C_{J, k+1,1,-1}^{J, k} P_{J}^{k+1}(\cos \theta) e^{i(k+1) \varphi} .
\end{gathered}
$$

Noting: (i) the complex azimuthal exponential terms $e^{i k \varphi}, e^{i m \varphi}$ in (2.18) and (4.33)-(4.34) (via the above representations for $B$ and $D$ ) for $|k| \leq J,|m| \leq L ; 1 \leq$ $L, J \leq N$, and (ii) the need to evaluate several $\mathcal{O}\left(N^{2}\right)$ sums, of the form in (4.24) and (4.28)-(4.29), at the equally spaced $\mathcal{O}(N)$ azimuthal quadrature points (see (3.6) ), we reduce the complexity by $\mathcal{O}(N)$ in each of these sums, at each adaptivetime step (described below), by using the FFT for setting up the nonlinear system (4.2), similar to the approach in 4. In our numerical experiments (see Section 5) for adaptive-time simulation of a flow induced by random initial states, we observed that such an efficient FFT based implementation reduced the (non-FFT code) computing time substantially for the case $N=100$, to simulate from $t=0$ to $t=60$.

In addition, by using the fast Legendre/spherical transforms along the latitudinal direction (obtained, for example, by modifying the NFFT algorithm in [21] for evaluation of the Legendre functions in the above terms at $\mathcal{O}(N)$ nonuniform latitudinal quadrature points), we could reduce the complexity by $\mathcal{O}\left(N^{2}\right)$. We did not use the fast Legendre/spherical transforms in our implementation due to the spectral convergence of the scheme and the fact that $N \leq 100$ in our simulations. (In these complexity counts, we ignored $\mathcal{O}(\log N)$ and $\mathcal{O}\left(\log ^{2} N\right)$ terms.)

\section{NumeriCAL EXPERIMENTS}

We demonstrate the fully discrete pseudospectral quadrature algorithm by simulating: (i) a known solution test case with low to high frequency modes and (ii) a benchmark example [8, page 305] in which the unknown velocity and vorticity fields are generated by a random initial state.

The first test example is useful to demonstrate that the pseudospectral quadrature algorithm reproduces any number of high frequency modes in the solution 
(provided $V_{N}$ contains all these modes), with computational error dominated only by the chosen accuracy for the adaptive time evolution for the ordinary differential system (4.25).

5.1. Example 1. Our first test case example is (2.1) with

$$
\begin{gathered}
\left.\mathbf{u}\right|_{t=0}(\widehat{\mathbf{x}})=\mathbf{u}_{0}(\widehat{\mathbf{x}})=g(0)\left[\mathbf{W}_{1}(\widehat{\mathbf{x}})-\mathbf{W}_{2}(\widehat{\mathbf{x}})\right], \\
g(t)=\nu e^{-t}[\sin (5 t)+\cos (10 t)], \\
\mathbf{W}_{1}=\mathbf{Z}_{1,0}+2 \Re\left(\mathbf{Z}_{1,1}\right), \mathbf{W}_{2}=\mathbf{Z}_{2,0}+2 \Re\left(\mathbf{Z}_{2,1}+\mathbf{Z}_{2,2}\right),
\end{gathered}
$$

where $\mathbf{Z}_{L, m}$ is given by (2.17), $\Re(\cdot)$ denotes the real-part function, and the external force $\mathbf{f}(\widehat{\mathbf{x}}, t)$ in $(2.1)$ is chosen so that

$$
\mathbf{u}(\widehat{\mathbf{x}}, t)=t g(t) \sum_{L=1}^{N_{0}}\left[\mathbf{Z}_{L, 0}+2 \sum_{m=1}^{L} \Re\left(\mathbf{Z}_{L, m}\right)\right](\widehat{\mathbf{x}})+g(t) \mathbf{W}_{1}(\widehat{\mathbf{x}})+(t-1) g(t) \mathbf{W}_{2}(\widehat{\mathbf{x}}),
$$

is the exact tangential divergence-free velocity field, solving the NSE (2.1). The exact test field (5.2)-(5.3) has high oscillations both in space and time, and exponentially decays in time. Note the dependence on a parameter $N_{0}$, the maximum order of the spherical harmonics in the exact solution.

In our calculation of the approximate solution $\mathbf{u}_{N}$, we chose $N=N_{0}$, so that all frequencies of the exact solution can be recovered. The solution (5.3) is then used to validate our algorithm and code by numerical adaptive time-integration of (4.1), for various values of $N=N_{0}$. In particular, for a fixed integration tolerance error, we demonstrate in Figure 1 that all $N$ modes in (5.3) can indeed be recovered by the approximate solution $\mathbf{u}_{N}$, within the chosen error tolerance, for all $N=N_{0}=$ $70,80,90,100$.

5.2. Example 2. Having established the validity of our algorithm for a simple known solution, we use the same code to simulate unknown velocity and vorticity fields generated by (2.1) with random initial velocity field as in [8, 12] with angular velocity of the rotation $\Omega=1$ and $\nu^{-1}=10,000$ in (2.1).

The initial flow and external force in this benchmark example satisfy the main assumption (3.1) for any $s, \sigma_{1}>0$ and hence, as proved in Theorem 4.2, the approximate solution $\mathbf{u}_{N}$ is spectrally accurate and converges super-algebraically with order given by (4.8) for any $s>0$. As mentioned in Section 1, this is the main advantage of the present paper over the recent paper [12, where such spectrally accurate convergence results are neither discussed nor proved. On the other hand, convergence results for two-dimensional problems on a Euclidean plane, supported by numerical experiments, have formed a core part of research on the NSE over the last few decades; see [8, 9, 27] and references therein.

The random initial tangential divergence-free velocity field, having properties similar to those considered in [8, page 305] and [12, page 988] (but not exactly the same as in [8, 12, due to randomness), is a smooth function $\mathbf{u}_{0}=\mathbf{v} \in G_{\sigma}^{s / 2}$, a Gevrey class of order $s$ and index $\sigma$ (see (2.33), for any $s, \sigma>0$ ), with Fourier coefficients $\widehat{\mathbf{v}}_{L, m}, L=1,2, \ldots,|m| \leq L$, defined by

$$
\widehat{\mathbf{v}}_{L, m}= \begin{cases}a_{L} \exp \left(i \phi_{m}\right) & L=1, \ldots, 20 ; m=0, \ldots, L, \\ a_{L}(-1)^{m} \exp \left(-i \phi_{m}\right) & L=1, \ldots, 20 ; m=-L, \ldots,-1, \\ 0, & L>20 ; m=-L, \ldots, L,\end{cases}
$$




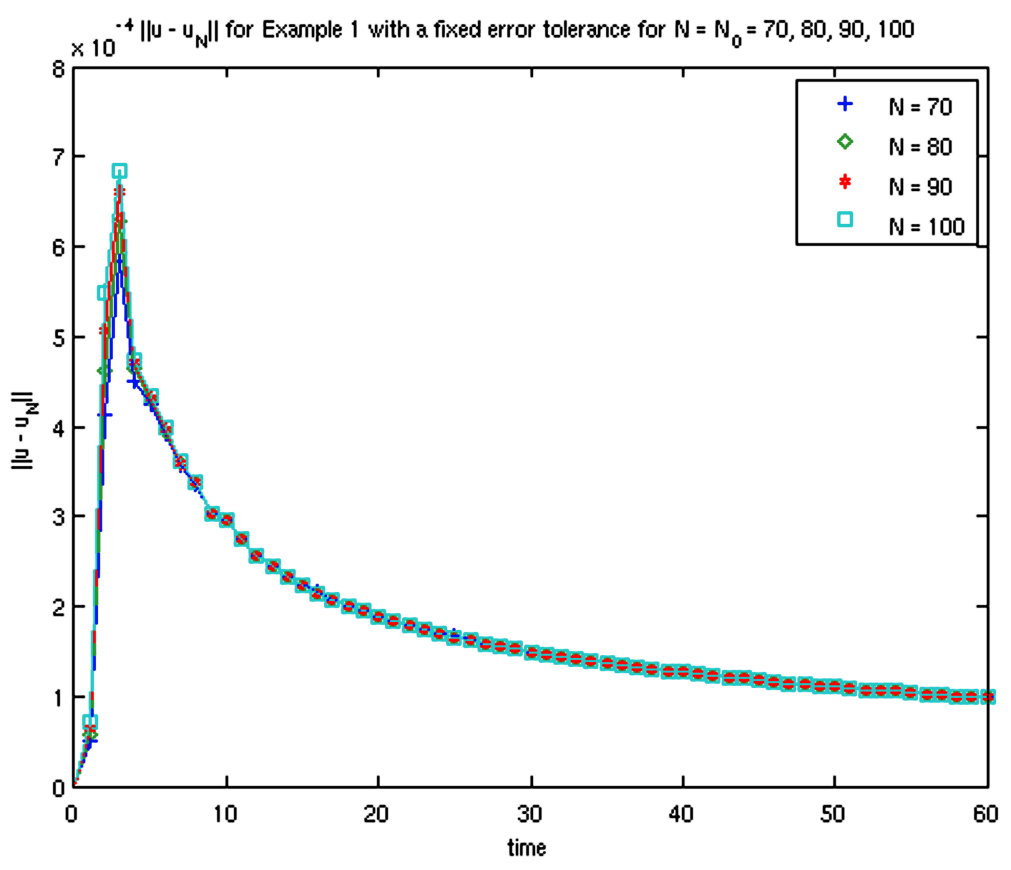

Figure 1. $\left\|\mathbf{u}-\mathbf{u}_{N}\right\|$ for Example 1 with a fixed time-integration error, $N=N_{0}=70,80,90,100$.

where $\phi_{0}=0$, and $\phi_{m} \in(0,2 \pi)$ are random numbers for $m>0$, and $a_{L}=b_{L} /\|\mathbf{b}\|$, with $\mathbf{b} \in \mathbb{R}^{20}$ having components $b_{L}=2 /\left[L+(\nu L)^{2.5}\right], L=1, \ldots, 20$. The vorticity stream function $\Psi$ (see (2.2) $)$ of $\operatorname{Vort} \mathbf{u}(\cdot, 0)$ in Figure 2 demonstrates the randomness of the field at time $t=0$.

The external force field $\mathbf{f}=\mathbf{f}(\widehat{\mathbf{x}}, t)$ in (2.1) for our simulation is motivated by that considered in [8, page 305] and is exactly same as that in [12, page 988]. The source term $\mathbf{f}$ is a decaying tangential divergence-free field which, for any $s, \sigma>0$, belongs to $C\left([0, T] ; \mathcal{D}\left(\mathbf{A}^{s+1 / 2} e^{\sigma \mathbf{A}^{1 / 2}}\right)\right)$ (for any $T>0$ ) with the only nonzero Fourier coefficient being $\widehat{\mathbf{f}(t)}_{3,0}$. The Fourier coefficient $\widehat{\mathbf{f}(t)}_{3,0}$ is a continuous function in time and is defined by

$$
\widehat{\mathbf{f}(t)}_{3,0}= \begin{cases}1, & 0 \leq t \leq 10 \\ \cos (\pi t / 5) \exp (-(t-10) / 5) & t>10\end{cases}
$$

The impact of the external force on the numerical velocity and its complement (generating the approximate inertial manifold) is demonstrated in Figure 3 with the time evolution of the velocity field matching that of the external force, leading to little change in the evolution process of the velocity as the external force gets smaller and smaller.

We chose a fixed relative error tolerance to be of accuracy at least $\mathcal{O}\left(10^{-3}\right)$, for adaptive time-integration solving the $N^{2}+2 N$-dimensional system ordinary differential equations (4.1) in time, using backward differential formulas with variable order (one to five) and variable adaptive time step sizes that meet the fixed error tolerance. 


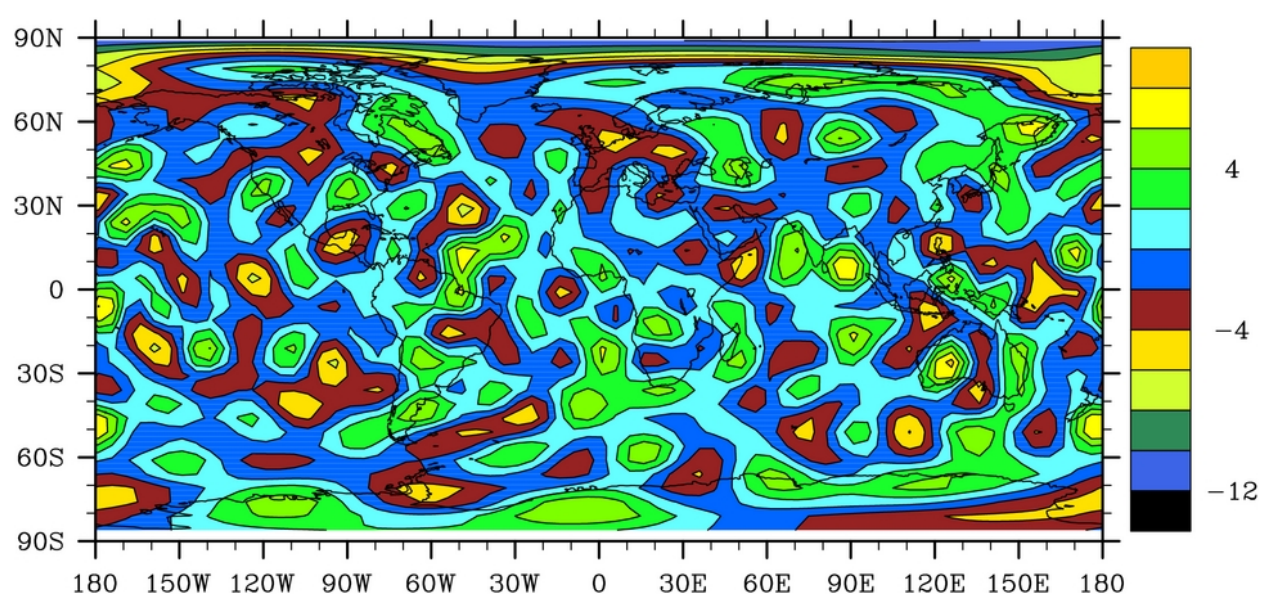

Figure 2. Initial random vorticity stream function $\Psi$ of Vort $\mathbf{u}$ at $t=0$.

As discussed in the next subsection, the high-frequency components of the solution turn out to be unreliable as $t$ increases, 8], presumably because of the time discretization error. We therefore retained only the frequency components up to some order $N_{1} \leq N$, correspondingly, we define an additional approximation of $\mathbf{u}_{N}$, defined in (4.1)-(4.24), by

$$
\mathbf{u}_{N_{1} ; N}(\cdot, t):=\Pi_{N_{1}} \mathbf{u}_{N}(\cdot, t), \quad N_{1} \leq N .
$$

As in Theorem 4.2, for the spatially discrete pseudospectral quadrature method, assuming (3.1) and exact time integration of (4.1), and hence using (2.35), (3.4), and (4.8), we get spectral convergence:

$\left\|\mathbf{u}-\mathbf{u}_{N_{1} ; N}\right\| \leq\left\|\mathbf{u}-\boldsymbol{\Pi}_{N_{1}} \mathbf{u}\right\|+\left\|\boldsymbol{\Pi}_{N_{1}}\left(\mathbf{u}-\mathbf{u}_{N}\right)\right\| \leq C\left[N_{1}^{-2(s+1)}+N^{-2 s}\right] \leq C N_{1}^{-2 s}$.

Our simulated approximate velocity fields,

$$
\mathbf{U}_{75}(t):=\mathbf{u}_{75 ; 100}(t), \quad \text { for } t=10,20,30,40,50,60,
$$

are in Figures 49 and the associated vorticity stream function $\Psi_{75}(t)$ of Vort $\mathbf{U}_{75}(t)$ (computed using (4.32) ) are in Figures 10,15. These figures demonstrate that the initial random flow with several smaller structures evolve into regular flow with larger structures, similar to those observed in [8, page 307]. The choice of $N_{1}$ will be discussed in the next subsection.

5.3. Energy spectrum of the solution. If $\mathbf{u}$ (and hence the number of modes in $\mathbf{u}$ ) is unknown, as it is in the case in the benchmark test, Example 2, and if $\mathbf{u}_{N}$ does not contain all of the modes in $\mathbf{u}$, the higher modes of $\mathbf{u}_{N}$ are usually less accurate than the chosen practical error tolerance and hence can even violate important physical properties of $\mathbf{u}$ (because of the error time-integration tolerance being not very small). In such cases, it is important to choose $N_{1}<N$, depending on certain known physical properties of $\mathbf{u}$. 
For a fixed time $t$, the $L$-th mode energy spectrum of a tangential divergence-free flow $\mathbf{u}$ on the sphere is defined by

$$
\begin{aligned}
E(L) & =E(\mathbf{u}, L)=\sum_{|m| \leq L}\left|\beta_{L, m}(t)\right|^{2}, \\
\mathbf{u}(\widehat{\mathbf{x}}, t) & :=\sum_{L=1}^{\infty} \sum_{|m| \leq L} \beta_{L, m}(t) \mathbf{Z}_{L, m}(\widehat{\mathbf{x}}) .
\end{aligned}
$$

Although the analytical form of the flow in Example 2 is not known, several investigations have been carried out for such fields with initial spectrum of the $L$-th mode decaying with order $L^{-1}$ or $L^{-2}$. In particular, it is well known (see [8]), for this benchmark test case (on periodic two-dimensional geometries), that the energy spectrum of the velocity has a power-law inertial range and an exponential decay (dissipation range) for wave numbers larger than the Kraichnan's dissipation wave number. Further, several random smaller structures built in the initial random vorticity evolve into regular flow with larger structures.

With $\mathbf{u}$ being the unique solution of the NSE (2.1), let us decompose $\mathbf{u}=$ $\widetilde{\mathbf{u}}_{N_{1}}+\mathbf{w}_{N_{1}}$, where $\widetilde{\mathbf{u}}_{N_{1}}=\boldsymbol{\Pi}_{N_{1}}(\mathbf{u})$ contains all modes lower or equal $N_{1}$ and $\mathbf{w}_{N_{1}}:=$ $\mathbf{u}-\boldsymbol{\Pi}_{N_{1}}(\mathbf{u})$ contains all higher modes. The existence of a relation between $\mathbf{w}_{N_{1}}$ and $\widetilde{\mathbf{u}}_{N_{1}}$ of a form $\mathbf{w}_{N_{1}}=\Phi\left(\widetilde{\mathbf{u}}_{N_{1}}\right)$ was established in [28. The graph of $\Phi$ is known as the inertial manifold of (2.1). For computational purposes, the higher modes can be computed efficiently using an approximate inertial manifold:

$$
\widetilde{\Phi}_{\widetilde{N}_{1}}\left(\widetilde{\mathbf{u}}_{N_{1}}\right)=(\nu \mathbf{A}+\mathbf{C})^{-1}\left(\boldsymbol{\Pi}_{\widetilde{N}_{1}}-\boldsymbol{\Pi}_{N_{1}}\right)\left[\mathbf{f}-\mathbf{B}\left(\widetilde{\mathbf{u}}_{N_{1}}, \widetilde{\mathbf{u}}_{N_{1}}\right)\right], \widetilde{N}_{1}>N_{1}
$$

This well-known approximation (without the Coriolis term) was introduced in [10, 11. for nonlinear dissipative systems, including the NSE on domains and we choose $\widetilde{N_{1}}=2 N_{1}$.

For the benchmark test case, in the dissipation range, even $L^{4} E\left(\widetilde{\Phi}_{2 N_{1}}\left(\widetilde{\mathbf{u}}_{N_{1}}\right), L\right)$ decays exponentially to zero, further justifying restriction of the infinite dimensional range after certain values of $N_{1}$. As discussed in [8, pages 280 and 307] (and repeated in [12, page 991]), such a faster decay (one order higher than the $L^{-3}$ decay known is Kraichnan theory of turbulence) is expected due to the Reynolds number considered in 8, 12 being much smaller than 25,000. (Extensive studies in [3, 23. show that the turbulence theory decay can occur only when the Reynolds number is of the order 25,000.)

Briefly (without repeating technical details in 8), using the viscosity term $\nu$ in (2.1), the Reynolds number is $\mathcal{O}\left(\nu^{-1}\right)$ with the order constant $r$ given by the product of the mean fluid velocity and the characteristic length-scale. With $\nu^{-1}=$ 10,000 and the constant rotation rate $\Omega=1$, for the Coriolis parameter in (2.23), the decay of energy spectrum of a numerical velocity and exponential decay of its approximate complement in Figures 16 and 17 is well supported by extensive simulations in $[3,23,8$, highlighting further the benchmark applicability of our algorithm, extending periodic domain results in [3, 23, 8, to a practically relevant rotating sphere case with Coriolis effect. 
Further, the simulated results are substantiated by the well-known exponential decay of $E\left(\widetilde{\Phi}_{2 N_{1}}\left(\widetilde{\mathbf{u}}_{N_{1}}\right), L\right)$, observed in Figures [16 and 17] Finally, the exponential decay of the energy spectrum show that $N=100, N_{1}=3 N / 4$ is sufficient to understand the flow behavior for this benchmark example with our algorithm using adaptive variable order and variable time-step highly stable backward differentiation formulas with a practically useful relative error tolerance $\mathcal{O}\left(10^{-3}\right)$.
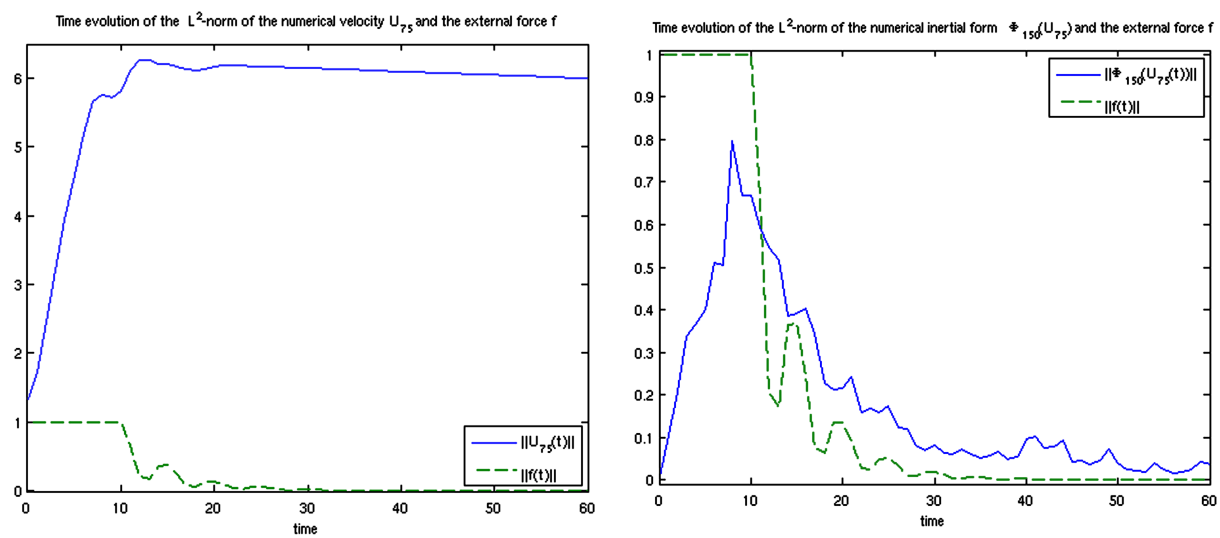

Time evolution of $\mathbf{U}_{75}(t)$ and $f(t)$.

Time evolution of $\widetilde{\Phi}_{150}\left(\mathbf{U}_{75}(t)\right)$ and $\mathbf{f}(t)$.

Figure 3. Impact of the external force on a numerical velocity and its complement.

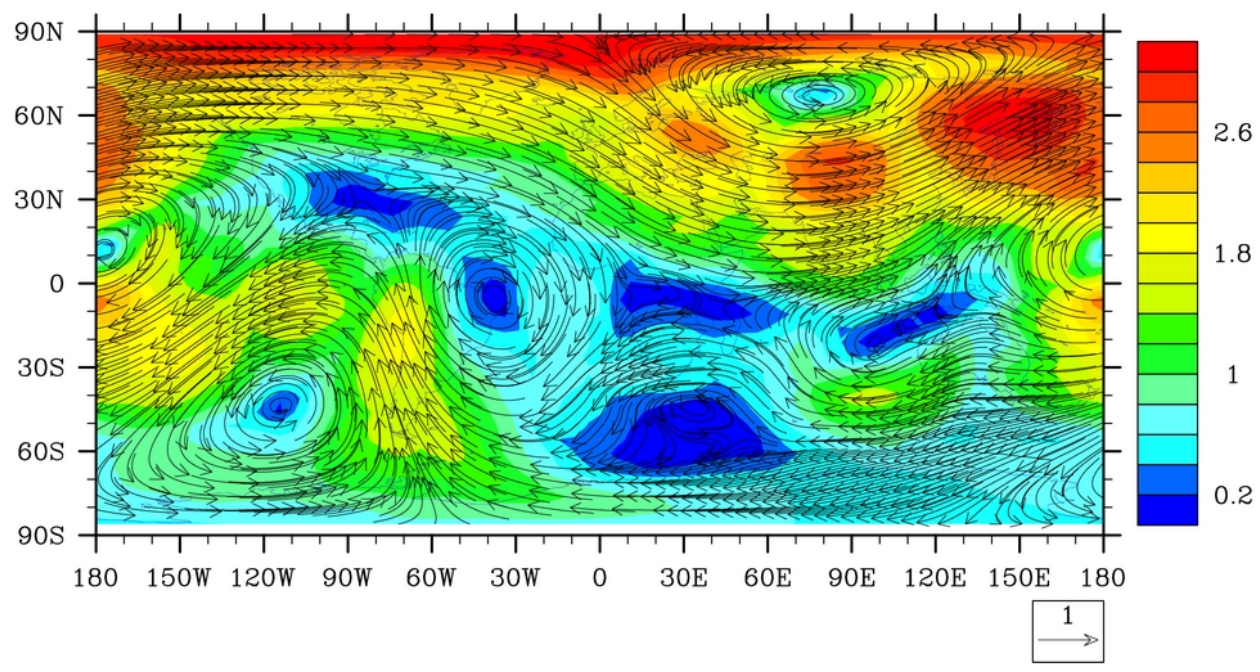

Figure 4. Numerical velocity $\mathbf{U}_{75}(t)$, at $t=10$. 


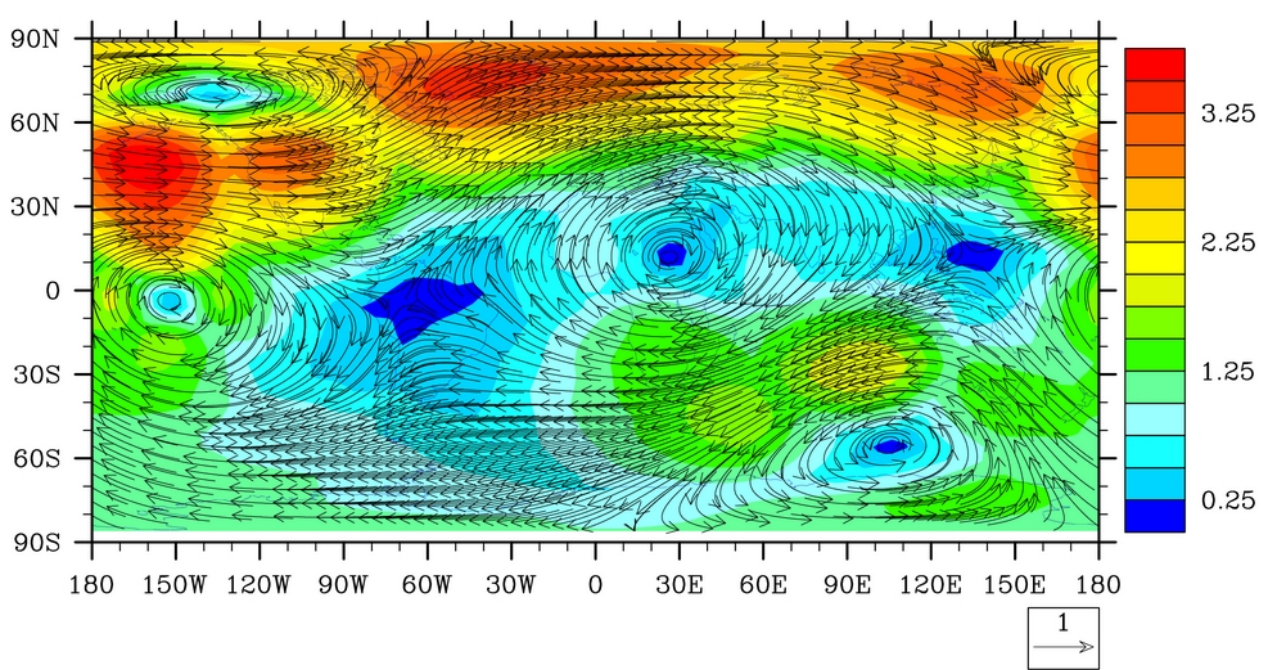

Figure 5. Numerical velocity $\mathbf{U}_{75}(t)$, at $t=20$.

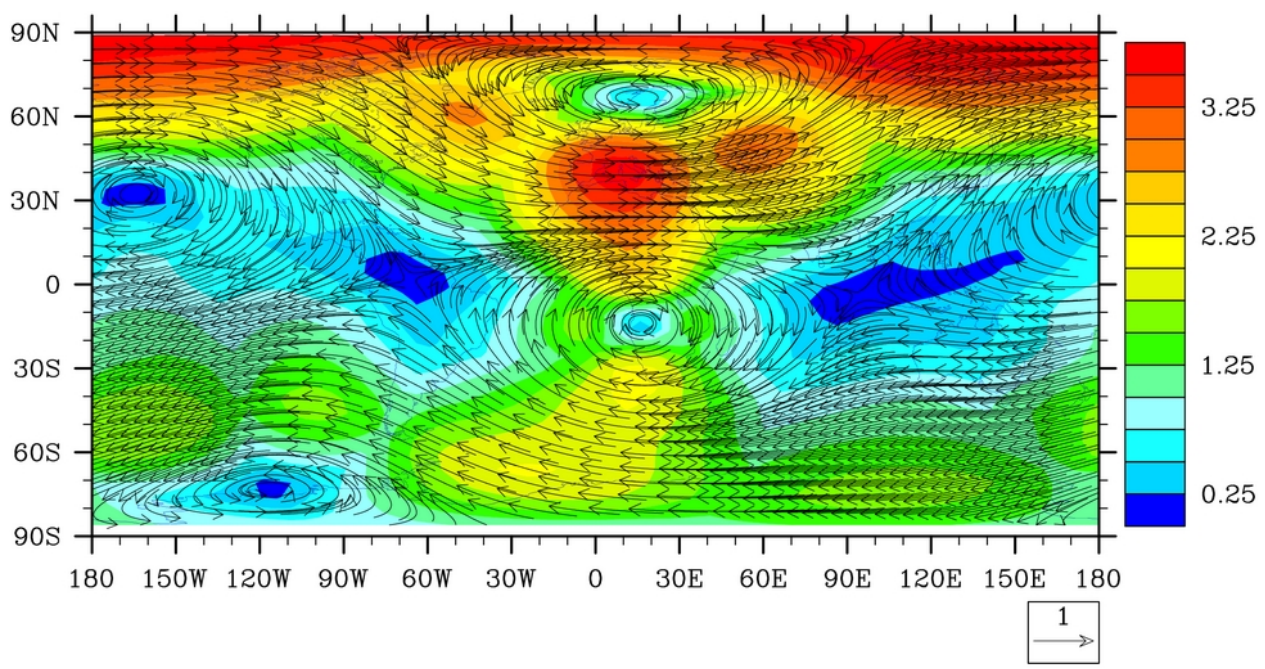

Figure 6 . Numerical velocity $\mathbf{U}_{75}(t)$, at $t=30$. 


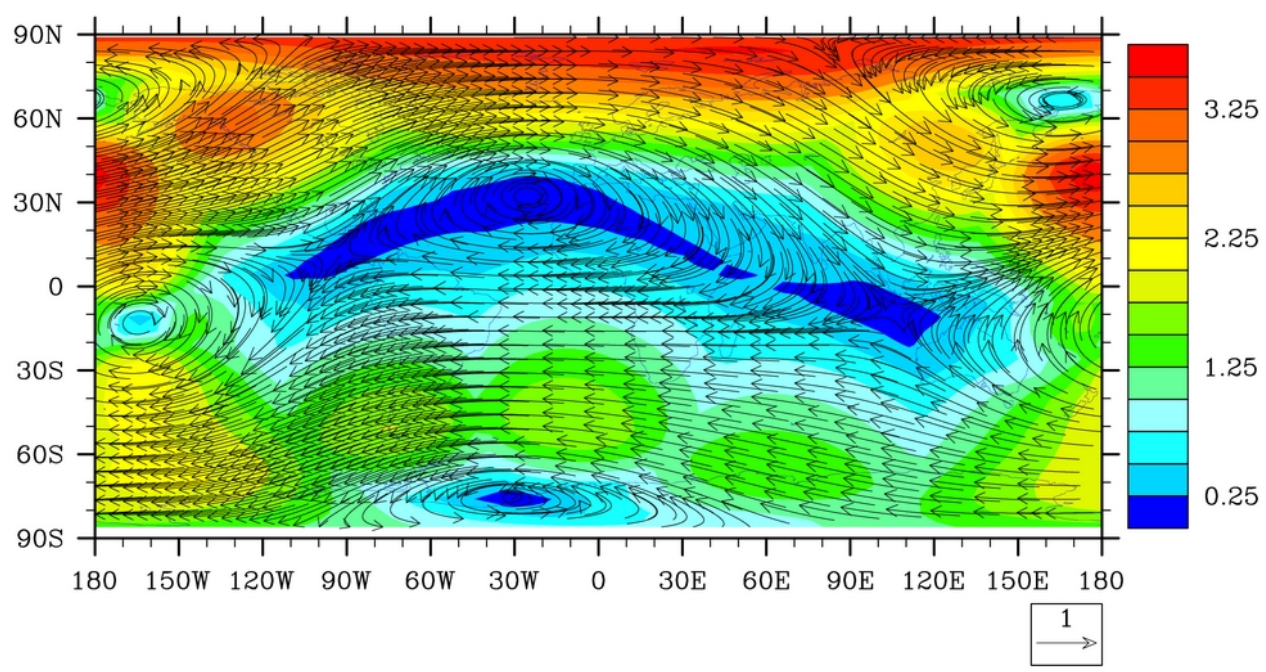

Figure 7 . Numerical velocity $\mathbf{U}_{75}(t)$, at $t=40$.

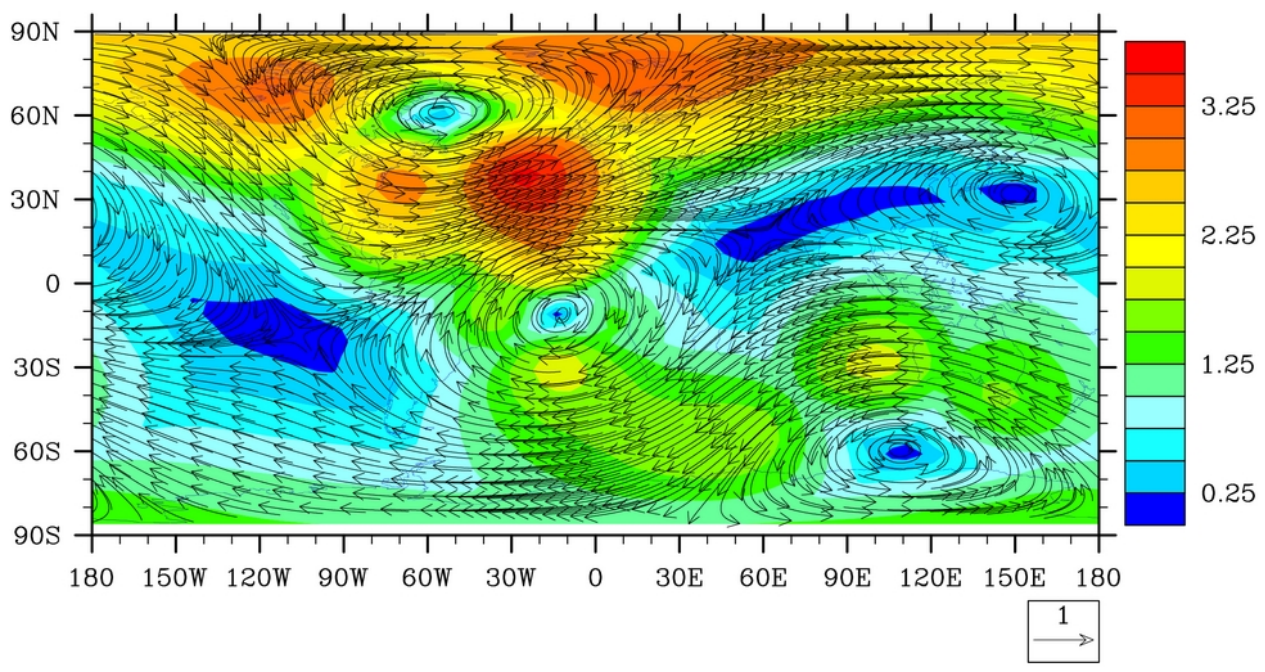

Figure 8. Numerical velocity $\mathbf{U}_{75}(t)$, at $t=50$. 


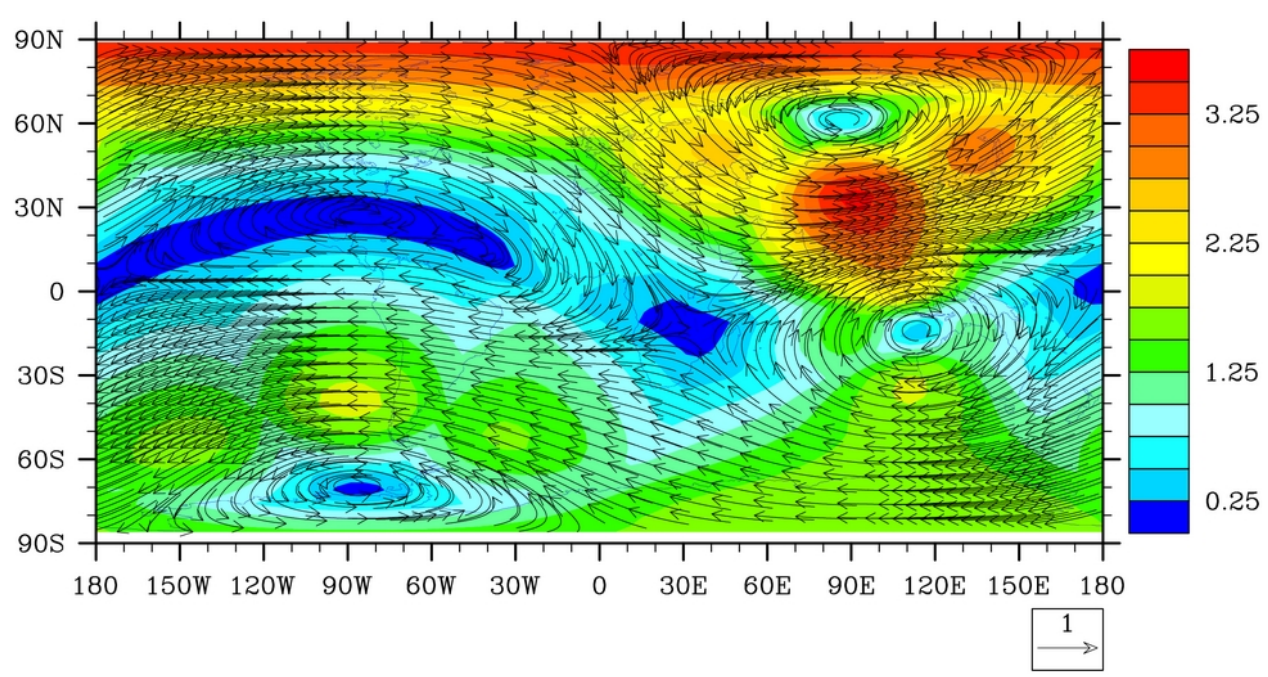

Figure 9. Numerical velocity $\mathbf{U}_{75}(t)$, at $t=60$.

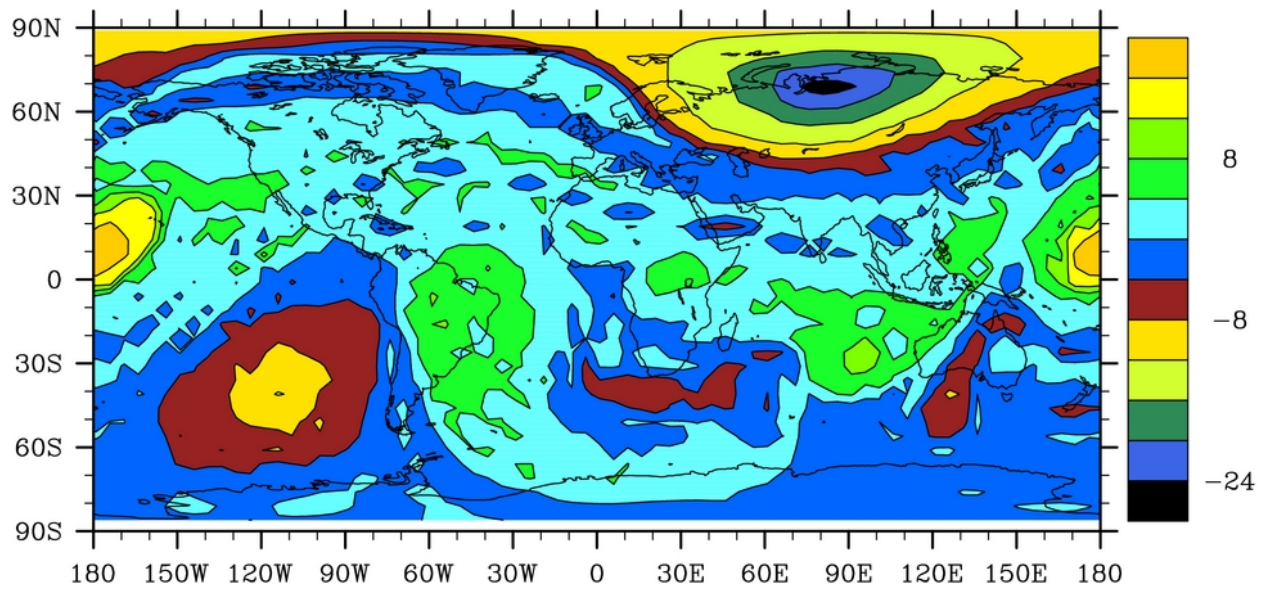

Figure 10. Vorticity stream function $\Psi_{75}$ of Vort $\mathbf{U}_{75}$ at $t=10$. 


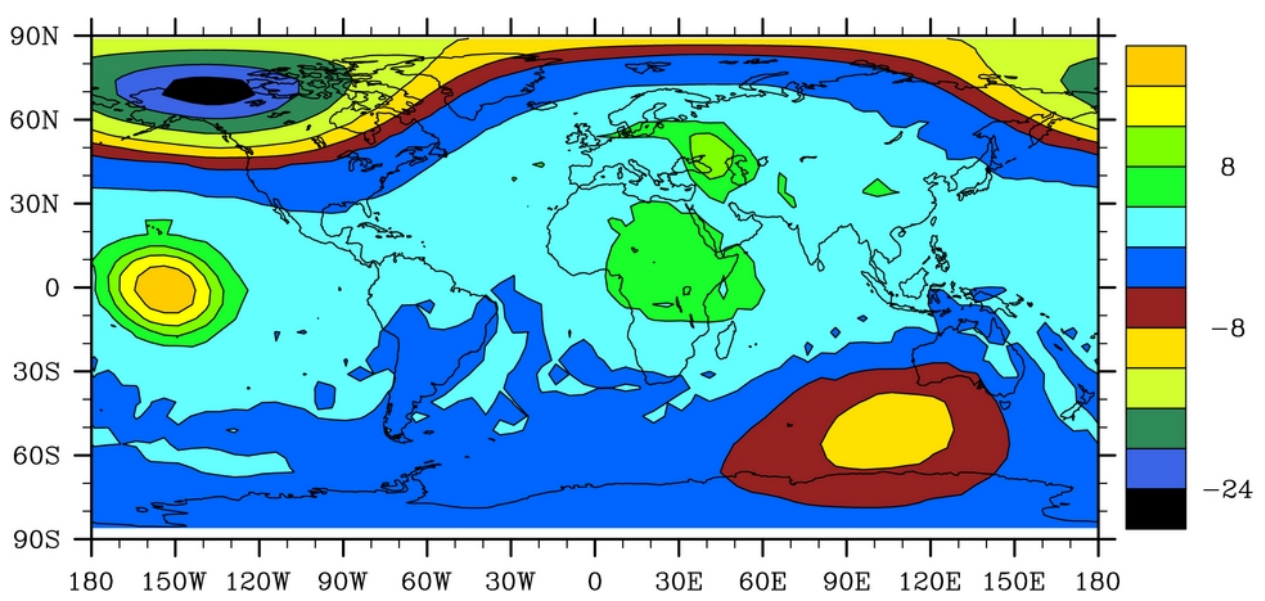

Figure 11. Vorticity stream function $\Psi_{75}$ of Vort $\mathbf{U}_{75}$ at $t=20$.

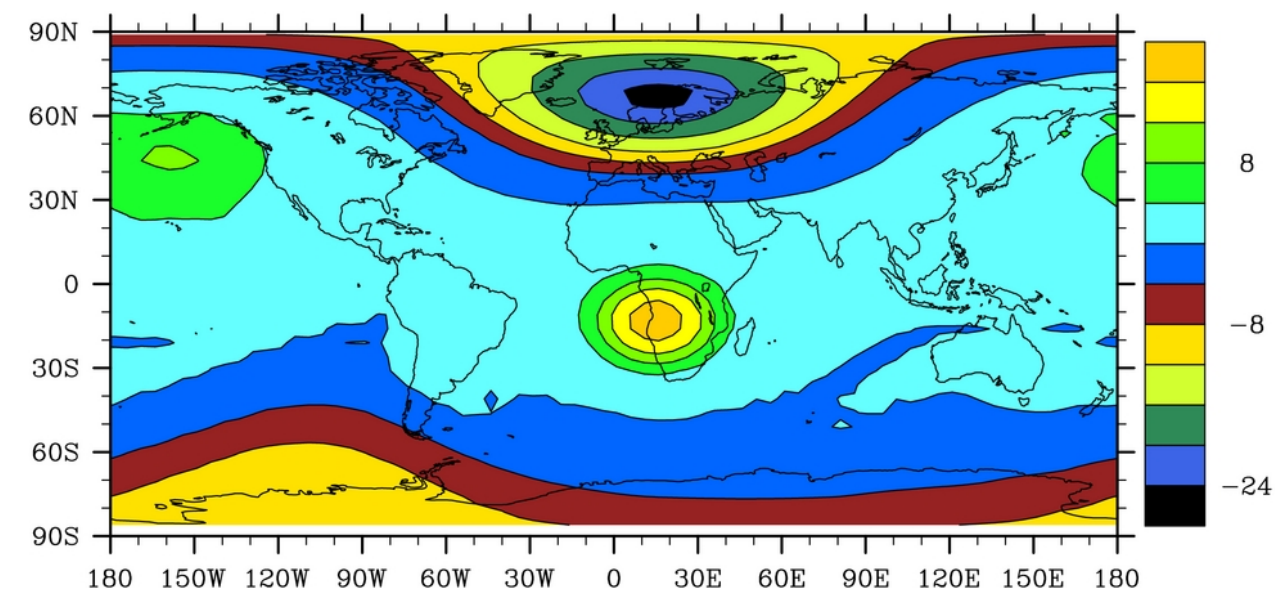

FiguRE 12. Vorticity stream function $\Psi_{75}$ of Vort $\mathbf{U}_{75}$ at $t=30$. 


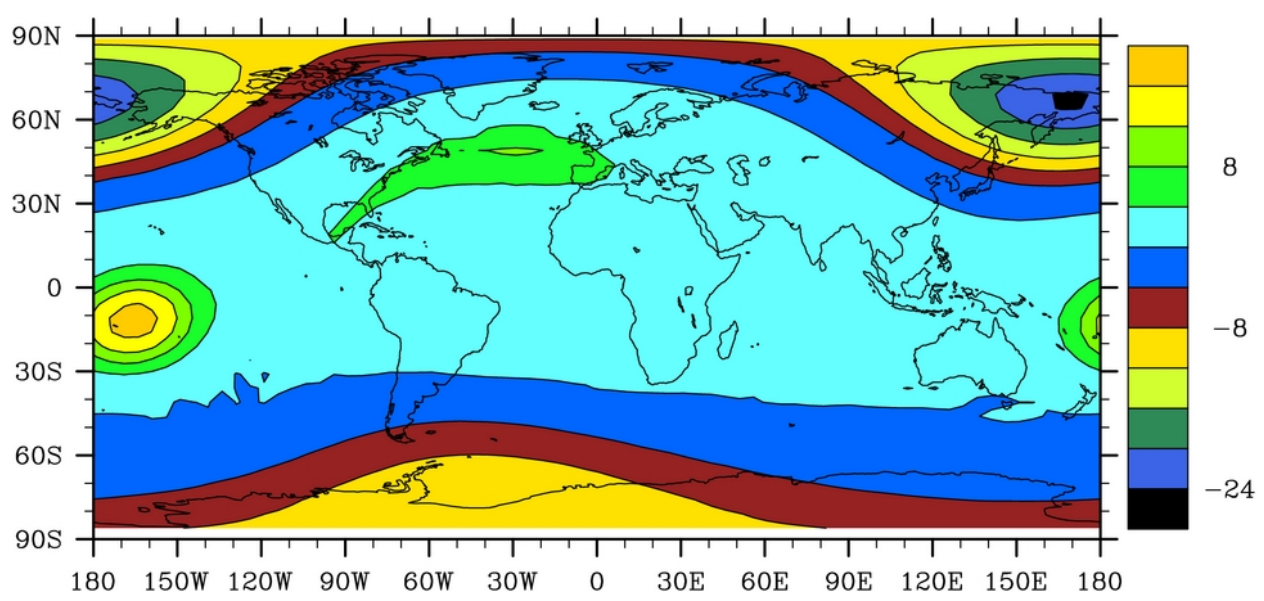

Figure 13. Vorticity stream function $\Psi_{75}$ of Vort $\mathbf{U}_{75}$ at $t=40$.

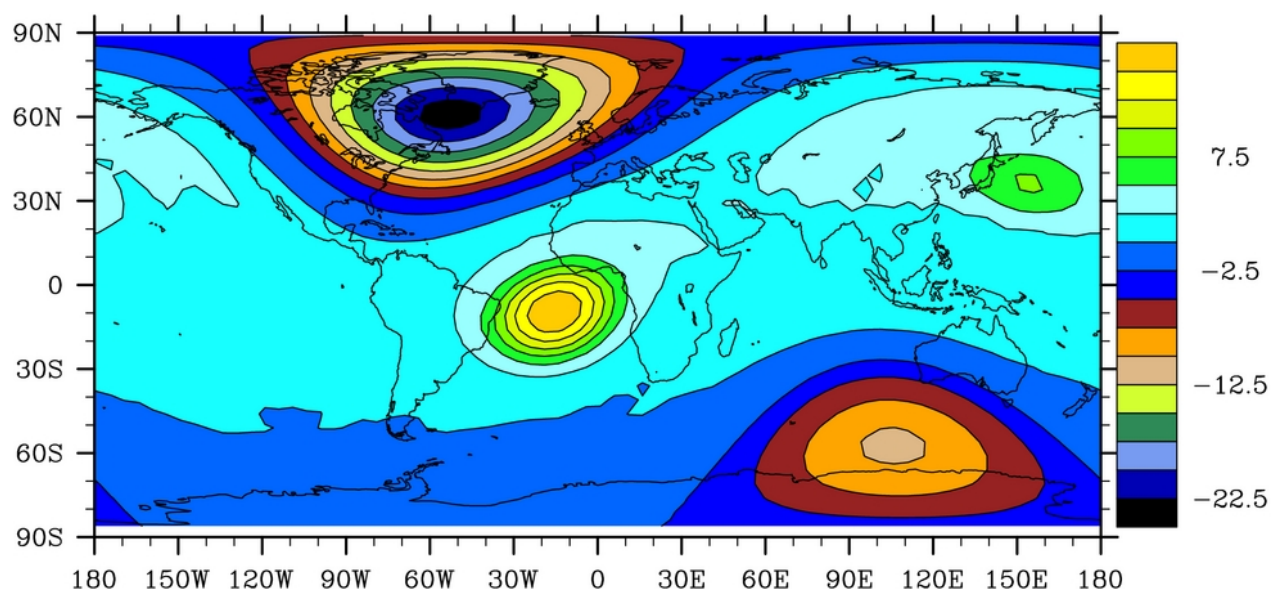

FiguRE 14. Vorticity stream function $\Psi_{75}$ of Vort $\mathbf{U}_{75}$ at $t=50$. 


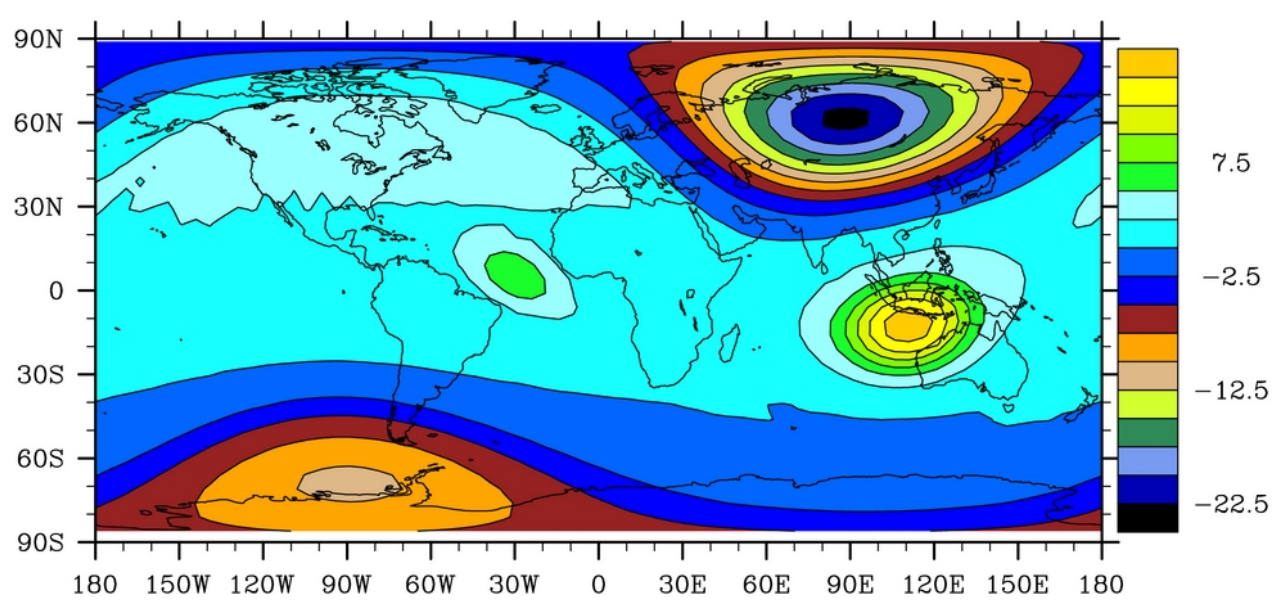

Figure 15. Vorticity stream function $\Psi_{75}$ of Vort $\mathbf{U}_{75}$ at $t=60$.

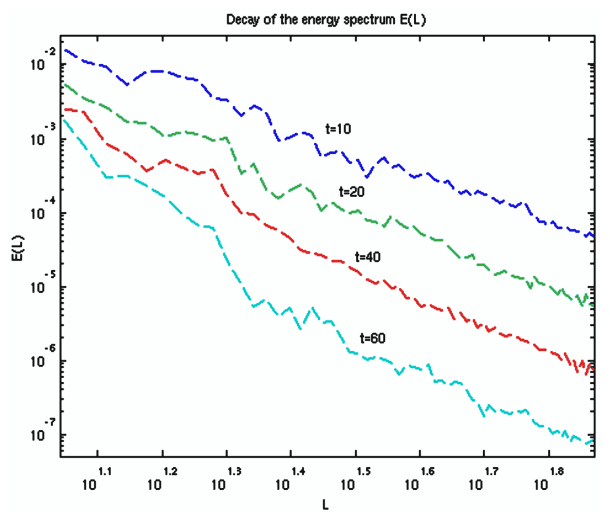

Energy spectrum $E(L)$ of $\mathbf{U}_{75}(t)$.

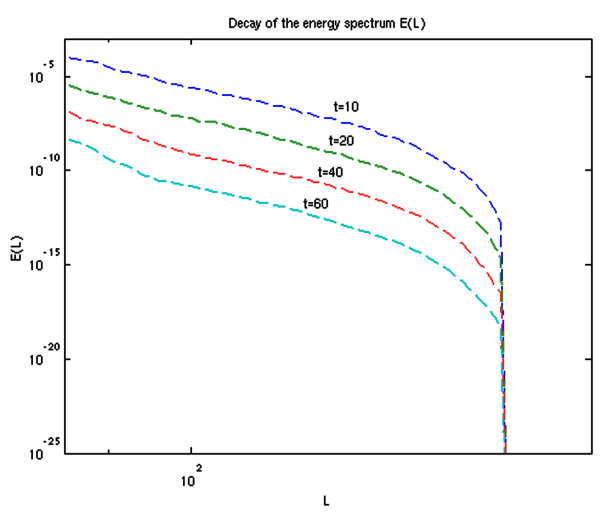

Energy spectrum $E(L)$ of $\widetilde{\Phi}_{150}\left(\mathbf{U}_{75}(t)\right)$.

FIGURE 16. Energy spectra of velocity $\mathbf{U}_{75}(t)$ and $\Phi_{150}\left(\mathbf{U}_{75}(t)\right)$. 


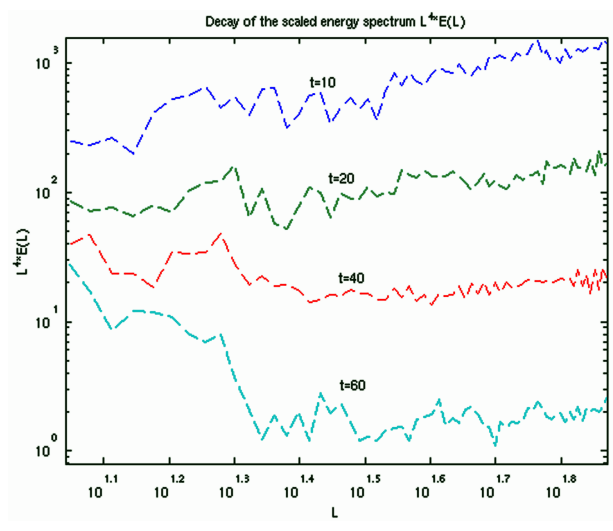

Energy spectrum $L^{4} * E(L)$ of $\mathbf{U}_{75}(t)$

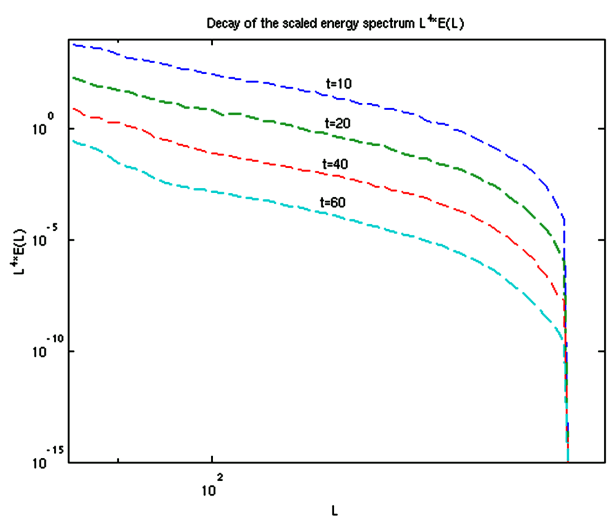

Energy spectrum $L^{4} * E(L)$ of $\widetilde{\Phi}_{150}\left(\mathbf{U}_{75}(t)\right)$

Figure 17. Scaled energy spectra $L^{4} * E(L)$ of $\mathbf{U}_{75}(t)$ and $\widetilde{\Phi}_{150}\left(\mathbf{U}_{75}(t)\right)$.

\section{ApPEndix}

In this section, we generalize certain known domain case estimates, that are fundamental for the NSE analysis, to the spherical surface case and hence prove (2.30). Following [16, Page 574], for $\mathbf{u} \in C^{\infty}(T S)$, we extend $\mathbf{u}$ to the spherical layer $S \times I, I=\left(r_{1}, r_{2}\right), 0<r_{1}<1<r_{2}<\infty$ by the formula

$$
\widetilde{\mathbf{u}}(\widehat{\mathbf{x}})=\varphi(|\widehat{\mathbf{x}}|) \mathbf{u}(\widehat{\mathbf{x}} /|\widehat{\mathbf{x}}|),
$$

where $\varphi(t) \in C_{0}^{\infty}(I), \varphi(t) \geq 0, t \in I$, and $\varphi(1)=1$. We have

$$
\int_{S \times I}|\widetilde{\mathbf{u}}|^{p}=\int_{r_{1}}^{r_{2}} \varphi^{p}(r) \int_{S}|\mathbf{u}|^{p} d S
$$

In other words,

$$
\|\widetilde{\mathbf{u}}\|_{L^{p}(S \times I)}=c\|\mathbf{u}\|_{L^{p}(T S)},
$$

where $c=c\left(\varphi, r_{1}, r_{2}\right)$. Suppose $\mathbf{u}, \mathbf{v}, \mathbf{w}$ are extended from $S$ to the spherical layer $S \times I$ by (6.1). Then from [16, Lemma 4.3] we have

$$
b(\mathbf{u}, \mathbf{v}, \mathbf{w})=c b(\widetilde{\mathbf{u}}, \widetilde{\mathbf{v}}, \widetilde{\mathbf{w}}),
$$

On the sphere $S$, we have the following version of Sobolev embedding inequality [1]

$$
\|\mathbf{u}\|_{L^{q}(T S)} \leq C\|\mathbf{u}\|_{H^{s}(T S)}, \quad s<1, \quad \frac{1}{q}=\frac{1}{2}-\frac{s}{2} .
$$

The following nonlinearity estimate is an adaptation of [6, Proposition 6.1] for $S$.

Proposition 6.1. Let $s_{1}, s_{2}, s_{3} \geq 0$ be real numbers, and we assume that $s_{1}+s_{2}+$ $s_{3} \geq 1$ and $\left(s_{1}, s_{2}, s_{3}\right) \neq(0,0,1),(0,1,0),(1,0,0)$. Then there exists a constant depending on $s_{1}, s_{2}, s_{3}$ such that

$$
|b(\mathbf{u}, \mathbf{v}, \mathbf{w})| \leq C\|\mathbf{u}\|_{H^{s_{1}(T S)}}\|\mathbf{v}\|_{H^{s_{2}+1}(T S)}\|\mathbf{w}\|_{H^{s_{3}(T S)}}
$$


or in the extrapolated form, writing $H^{s}(T S)=H_{T S}^{s}$, for all $\mathbf{u}, \mathbf{v}, \mathbf{w} \in C^{\infty}(T S)$,

$$
\begin{aligned}
& |b(\mathbf{u}, \mathbf{v}, \mathbf{w})| \\
& \quad \leq C\|\mathbf{u}\|_{H_{T S}^{\left[s_{1}\right]}}^{1+\left[s_{1}\right]-s_{1}}\|\mathbf{u}\|_{H_{T S}^{\left[s_{1}\right]+1}}^{s_{1}-\left[s_{1}\right]}\|\mathbf{v}\|_{H_{T S}^{\left[s_{2}\right]+1}}^{1+\left[s_{2}\right]-s_{2}}\|\mathbf{v}\|_{H_{T S}^{\left[s_{2}\right]+2}}^{s_{2}-\left[s_{2}\right]}\|\mathbf{w}\|_{H_{T S}^{\left[s_{3}\right]}}^{1+\left[s_{3}\right]-s_{3}}\|\mathbf{w}\|_{H_{T S}^{\left[s_{3}\right]+1}}^{s_{3}-\left[s_{3}\right]} .
\end{aligned}
$$

Proof. Let $\mathbf{u}, \mathbf{v}, \mathbf{w} \in C^{\infty}(T S)$ and $\widetilde{\mathbf{u}}, \widetilde{\mathbf{v}}, \widetilde{\mathbf{w}}$ be their corresponding extension to the spherical layer $\widetilde{\Omega}:=S \times I$. Let us consider first the case $s_{i}<1$ for $i=1,2,3$. Define associated constants $q_{1}, q_{2}, q_{2}, q_{4}$ so that $\sum_{i=1}^{4} \frac{1}{q_{i}}=1$ and $\frac{1}{q_{i}}=\frac{1}{2}-\frac{s_{i}}{2}$ for $i=1,2,3$. Then, by Hölder's inequality we get

$$
|b(\widetilde{\mathbf{u}}, \widetilde{\mathbf{v}}, \widetilde{\mathbf{w}})|=\left|\sum_{i, j=1}^{3} \int_{\widetilde{\Omega}} \widetilde{u}_{j} \frac{\partial \widetilde{v}_{i}}{\partial x_{j}} \widetilde{w}_{i} 1\right| \leq\|\widetilde{\mathbf{u}}\|_{L^{q_{1}}(\Omega)}\|\nabla \widetilde{\mathbf{v}}\|_{L^{q_{2}(\Omega)}}\|\widetilde{\mathbf{w}}\|_{L^{q_{3}}(\Omega)}\|1\|_{L^{q_{4}}(\Omega)} .
$$

Restricting to the sphere by (6.3) and (6.2) then using the Sobolev embedding theorem on the sphere (6.4) we have

$|b(\mathbf{u}, \mathbf{v}, \mathbf{w})| \leq C\|\mathbf{u}\|_{L_{T S}^{q_{1}}}\|\mathbf{G r a d} \mathbf{v}\|_{L_{T S}^{q_{2}}}\|\mathbf{w}\|_{L_{T S}^{q_{3}}} \leq C\|\mathbf{u}\|_{H_{T S}^{s_{1}}}\|\mathbf{G r a d} \mathbf{v}\|_{H_{T S}^{s_{2}}}\|\mathbf{w}\|_{H_{T S}^{s_{3}}}$.

Lemma 6.1. Let $\delta \in(1 / 2,1)$ be given and $\mathbf{u}, \mathbf{v} \in V$. Then there exists $C$, independent of $\mathbf{u}$ and $\mathbf{v}$, such that

$$
\left\|\mathbf{A}^{-\delta} \mathbf{B}(\mathbf{u}, \mathbf{v})\right\| \leq C\left\{\begin{array}{l}
\left\|\mathbf{A}^{1-\delta} \mathbf{u}\right\|\|\mathbf{v}\| \\
\|\mathbf{u}\|\left\|\mathbf{A}^{1-\delta} \mathbf{v}\right\|
\end{array}, \quad \mathbf{u}, \mathbf{v} \in V .\right.
$$

Proof. Let $\mathbf{u}, \mathbf{v}, \mathbf{w} \in V$. Hence from (2.26), and by using Proposition 6.1 with $s_{1}=0, s_{2}=2 \delta-1>0$ and $s_{3}=2-2 \delta>0$,

$$
\left|b\left(\mathbf{u}, \mathbf{v}, \mathbf{A}^{-\delta} \mathbf{w}\right)\right|=\left|b\left(\mathbf{u}, \mathbf{A}^{-\delta} \mathbf{w}, \mathbf{v}\right)\right| \leq C\|\mathbf{u}\|\left\|\mathbf{A}^{-\delta} \mathbf{w}\right\|_{H^{2 \delta}(T S)}\|\mathbf{v}\|_{H^{2-2 \delta}(T S)} .
$$

Since $\left\|\mathbf{A}^{-\delta} \mathbf{w}\right\|_{H^{2 \delta}(T S)}=\left\|\mathbf{A}^{\delta} \mathbf{A}^{-\delta} \mathbf{w}\right\|=\|\mathbf{w}\|$, we get

$$
\left|b\left(\mathbf{u}, \mathbf{v}, \mathbf{A}^{-\delta} \mathbf{w}\right)\right| \leq C\|\mathbf{u}\|\|\mathbf{w}\|\|\mathbf{v}\|_{H^{2-2 \delta}(T S)}, \quad \mathbf{w} \in V .
$$

Since the inequality is true for all $\mathbf{w} \in V$, we obtain the first bound,

$$
\left\|\mathbf{A}^{-\delta} \mathbf{B}(\mathbf{u}, \mathbf{v})\right\| \leq C\|\mathbf{u}\|\|\mathbf{v}\|_{H^{2-2 \delta}(T S)}=C\|\mathbf{u}\|\left\|\mathbf{A}^{1-\delta} \mathbf{v}\right\| .
$$

To obtain the second bound, we again use (2.26) and Proposition 6.1 but with $s_{1}=2-2 \delta, s_{2}=2 \delta-1$ and $s_{3}=0$,

$$
\begin{aligned}
\left|b\left(\mathbf{u}, \mathbf{v}, \mathbf{A}^{-\delta} \mathbf{w}\right)\right|=\left|b\left(\mathbf{u}, \mathbf{A}^{-\delta} \mathbf{w}, \mathbf{v}\right)\right| & \leq C\|\mathbf{u}\|_{H^{2-2 \delta}(T S)}\left\|\mathbf{A}^{-\delta} \mathbf{w}\right\|_{H^{2 \delta}(T S)}\|\mathbf{v}\| \\
& =C\left\|\mathbf{A}^{1-\delta} \mathbf{u}\right\|\|\mathbf{w}\|\|\mathbf{v}\|, \quad \mathbf{w} \in V .
\end{aligned}
$$

Since the inequality is true for all $\mathbf{w} \in H$, we obtain

$$
\left\|\mathbf{A}^{-\delta} \mathbf{B}(\mathbf{u}, \mathbf{v})\right\| \leq C\left\|\mathbf{A}^{1-\delta} \mathbf{u}\right\|\|\mathbf{v}\| .
$$

\section{ACKNowledgments}

The support of the Australian Research Council under its Discovery and Centre of Excellence programs is gratefully acknowledged. The authors thank Professors M. Farge and E. S. Titi 29] for valuable discussions. 


\section{REFERENCES}

[1] T. Aubin, Nonlinear analysis on manifolds, Monge-Ampère Equations. Springer-Verlag, 1982. MR681859 (85j:58002)

[2] J.P. Boyd, Chebyshev and Fourier Spectral Methods, Dover, 2001. MR1874071 (2002k:65160)

[3] M.E. Brachet, M. Meneguzzi, H. Politano, P.L. Sulem, The dynamics of freely decaying twodimensional turbulence. J. Fluid Mech. 194 (1988), 333-349.

[4] M.A.J. Chaplain, M. Ganesh, I.G. Graham, Spatio-temporal pattern formation on spherical surfaces: Numerical simulation and application to solid tumour growth. J. Math. Biology 42 (2001), 387-423. MR1842835 (2002g:92005)

[5] C. Cao, M.A. Rammaha, E.S. Titi, The Navier-Stokes equations on the rotating 2-D sphere: Gevrey regularity and asymptotic degrees of freedom. Zeit. Ang. Math. Phys. 50 (1999), 341-360. MR:1697711 (2001c:76030)

[6] P. Constantin, C. Foias, Navier-Stokes equations, Chicago Lectures in Mathematics, The University of Chicago Press, 1988. MR.972259 (90b:35190)

[7] A. Doelman, E.S. Titi, Regularity of solutions and the convergence of the Galerkin method in the Ginzburg-Landau equation. Numer. Funct. Anal. Optim. 14 (1993), 299-321. MR1229659 (94i:35179)

[8] A. Debussche, T. Dubois, R. Temam, The nonlinear Galerkin method: A multiscale method applied to the simulation of homogeneous turbulent flows. Theor. Comp. Fluid Dyn. 7 (1995), $279-315$.

[9] C. Foias, O. Manley, R. Rosa, R. Temam, Navier-Stokes Equations and Turbulence. Cambridge University Press, 2001. MR1855030 (2003a:76001)

[10] C. Foias, M.S. Jolly, I.G. Kevrekidis, G.R. Sell, E.S. Titi, On the computation of inertial manifolds. Phys. Lett. A 131 (1988), 433-436. MR972615 (89k:65154)

[11] C. Foias, O. Manley, R. Temam, Modelling of the interaction of small and large eddies in two dimensional turbulence flows, RAIRO Modél. Math. Anal. Numér. 22 (1988), 93-118. MR.934703 (89h:76022)

[12] M.J. Fengler, W. Freeden, A nonlinear Galerkin scheme involving vector and tensor spherical harmonics for solving the incompressible Navier-Stokes equation on the sphere. SIAM J. Sci. Comp. 27 (2005), 967-994. MR2199916 (2007k:76034)

[13] M. Ganesh, K. Mustapha, A fully discrete $H^{1}$-Galerkin method with quadrature for nonlinear advection-diffusion-reaction equations. Numer. Algorithms, 43 (2006), 355-383. MR.2308464 (2008c:65257)

[14] E. Hairer, G. Wanner, Solving Ordinary Differential Equations II: Stiff and differentialalgebraic problems. Springer-Verlag, 1982. MR,1439506 (97m:65007)

[15] J. Hesthaven, S. Gottlieb, D. Gottlieb, Spectral Methods for Time-Dependent Problems. Cambridge University Press, 2007. MR2333926 (2008i:65223)

[16] A.A. Il'in, The Navier-Stokes and Euler equations on two-dimensional closed manifolds. Math. USSR Sbornik 69 (1991), 559-579. MR1055527 (91f:35210)

[17] A.A. Il'in, Partially dissipative semigroups generated by the Navier-Stokes system on twodimensional manifolds, and their attractors. Russian Acad. Sci. Sbornik Mathematics $\mathbf{7 8}$ (1994), 47-76. MR1211366 (94b:35211)

[18] A.A. Il'in, Navier-Stokes equations on the rotating sphere. A simple proof of the attractor dimension estimate. Nonlinearity 7 (1994), 31-39. MR.1260131 (95a:35110)

[19] A.A. Il'in, A.N. Filatov, On unique solvability of the Navier-Stokes equations on the twodimensional sphere. Soviet Math. Dokl. 38 (1989), 9-13. MR953596 (89k:35183)

[20] D.A. Jones, E.S. Titi, A remark on quasi-stationary approximate inertial manifold for the Navier-Stokes equations. SIAM J. Math. Anal. 25 (1994),894-914. MR.1271316 (95c:35193)

[21] S. Kunis, D. Potts, Fast spherical Fourier algorithms. J. Comp. Appl. Math. 161 (2003), 75-98. MR2018576 (2004k:65270)

[22] J. Norbury, I. Roulstone (Eds.), Large-Scale Atmosphere-Ocean Dynamics, Vol. 183 2, Cambridge University Press, 2002.

[23] S.A. Orszag, Turbulence and transition: A progress report. Proc. 5th Int. Conf. Numer. Meth. Fluids Dyn., Lecture Notes in Phys., Vol. 59, Springer, 32-51, 1977. MR0464912 (57:4831)

[24] M. Pieper, Vector hyperinterpolation on the sphere, J. Approx. Theory. 156 (2009), 173-186. MR2494551 
[25] L.F. Shampine, M.W. Reichlet The Matlab ODE suite. SIAM J. Sci. Comput., 18 (1997), 1-22. MR1433374 (97k:65307)

[26] T. Tao, Why global regularity for Navier-Stokes is hard. http://terrytao.wordpress.com/ 2007/03/18/why-global-regularity-for-navier-stokes-is-hard/.

[27] R. Temam, Navier-Stokes Equations, Theory and Numerical Analysis, Amer. Math. Soc., 2001. MR 1846644 (2002j:76001)

[28] R. Temam, S. Wang, Inertial forms of Navier-Stokes equations on the sphere. J. Funct. Anal. 117 (1993), 215-242. MR1240265 (94i:35163)

[29] E.S. Titi, Private communication, (2009).

[30] D.A. Varshalovich, A.N. Moskalev, V.K. Khersonskii, Quantum Theory of Angular Momentum, World Scientific, 1988. MR 1022665 (90j:81062)

Department of Mathematical and Computer Sciences, Colorado School of Mines, Golden, Colorado 80401

E-mail address: mganesh@mines.edu

School of Mathematics and Statistics, University of New South Wales, Sydney, NSW 2052, Australia

E-mail address: qlegia@unsw.edu.au

School of Mathematics and Statistics, University of New South Wales, Sydney, NSW 2052, Australia

E-mail address: i.sloan@unsw.edu.au 